This document is published in:

Information Fusion (2015). 22, 127-140.

DOI: http://dx.doi.org/10.1016/j.inffus.2014.06.004

(C) 2014 Elsevier B. V.

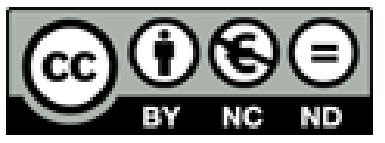

This work is licensed under a Creative Commons Attribution-NonCommercialNoDerivatives 4.0 International License. 


\title{
Model-based trajectory reconstruction with IMM smoothing and segmentation
}

\author{
Jesús García $^{a, *}$, Juan A. Besada ${ }^{b}$, José M. Molina ${ }^{a}$, Gonzalo de Miguel ${ }^{b}$ \\ a Computer Science Department-GIAA, Universidad Carlos III de Madrid, Spain \\ b Signal Systems and Radio-communications Department-GPDS, Universidad Politécnica de Madrid, Spain \\ * Corresponding author. Tel.: +34 676514284; fax: +34 918561271. E-mail address: jesus.garcia@uc3m.es (J. García).
}

\begin{abstract}
This paper presents a new approach for off-line trajectory reconstruction in air traffic control domain. The proposed algorithm, called model-based reconstruction, performs an accurate IMM smoothing process whose parameters are modified along time according to the flight modes segmented from trajectory measurements. Its competitive performance is demonstrated through comparison with previous reconstruction methods used in ATC and with classical IMM smoothing, using simulated data.
\end{abstract}

Keywords: Trajectory reconstruction, IMM data smoothing, ATC evaluation tools, MoF segmentation

\section{Introduction}

The air traffic control (ATC) domain is a critical sector placing high safety requirements for validation of tracking tools in operating conditions with real data sets. Performance assessment with live-recorded datasets (called opportunity traffic data) requires the reconstruction of references for evaluation [1]. These reference trajectories have to be reconstructed from the available data beforehand, since there is no ground truth available for comparison $[2,3]$. Then, the evaluation processes will compare a specific data source (both input sensors or tracker output at an ATC center) with these reconstructed references to obtain figures of merit (i.e. accuracy statistics, probabilities of detection, false alarm rates, etc). A representative example of assessment system based on opportunity traffic is SASS-C by Eurocontrol $[1,3,4]$. This suite contains different tools for analysis, displays, etc., to enable detailed analysis of system performance in real conditions.

Apart from the smoothed curve given as trajectory reference, the reconstruction process is required to provide kinematic information (velocity and accelerations) and the segmentation in predefined Modes of Flight (MoF) of the whole trajectory. The kinematic information is useful to derive the MoF and also to classify the target accordingly to magnitudes and motion patterns [5].

Historically, trajectory reconstruction has been performed using different mathematical methods. The first category is non-parametric, where a model-free learned system, like least squares, splines or neural networks, can be selected to approximate the real curve. Splines are the most common approach to fit data with non-parametric strategy [6-10] and reconstruction tools. For instance, a weighted least squares beta spline curve is calculated from the measurements in ATC domain in [3] or [11]. A recent analysis [12] also explains the use of splines in ATC domain. Such solutions do not use any knowledge of motion mod-els, but have the advantage of generality and robustness when accurate dynamic models are not available. Alternatively, if a para-metric representation can be used to model the trajectory then the available estimation methods can be used. Besides to ATC domain, totally similar ideas about trajectory reconstruction can be find in maritime environments $[13,14]$, robot movement on the ground [8,9] and unmanned vehicles [15].

Generally, the reconstruction problem can be formalized as a multisensor fusion process, equivalent to smoothing, as formulated in references [16,17]. In particular, it takes advantage of the availability of past and future observations to estimate the most accurate result. Theoretically, an optimal approach can be implemented by means of a double forward and backward tracking smoothing loop, known as the optimal smoother [17-20]. In the case of maneuvering targets, the combination of forward and backward IMM (Interacting Multiple Mode) filters have clear advantages over previous approaches based on maneuver detection [21], with difficulties to detect some transitions such as maneuvering to uniform motion mode. Ref. [22] reviews the state of the art in object tracking and multiple-model smoothing. A near optimal 
fixed interval smoothers based on IMM filters have been developed in $[20,22]$.

In this paper we present a new approach for ATC trajectory reconstruction $[23,11,12]$. The system uses an IMM-based smoother with variable model structure (the set of models change accordingly to the type of motion pattern, and also the dynamic and transition parameters). The data association and bias estimation processes, which are a critical factor in smoothing integrity, are assumed to be solved. This reconstruction algorithm is a central part of module named OTR (Opportunity Trajectory Reconstruction), which is in the core of current Eurocontrol SASS-C v7 system. It uses models of ATC trajectories to exploit predictable aircraft behavior in ATC reconstruction $[11,23,24]$. The method has three steps: (i) MoF trajectory segmentation based on the information obtained with two IMM filters, one on forward direction and other in backward, and some post-processing algorithms; (ii) Estimation of the set of models adapted to each MoF segment; (iii) Reconstruction using an IMM based optimal smoother.

Trajectory reconstruction based on MoF segmentation has been previously proposed in [3], and reconstruction in [18], used as reference for comparisons. Besides the use of IMM approach for jump Markov linear systems, other non-linear algorithms have been recently explored for maneuvering target tracking and fixed-lag smoothing. This is the case of the smoothing particle filter, a Bayesian filter to estimate recursively the posterior distribution. A variation from it, Rao-Blackwellized Particle Filter (RBPF), marginalizes some components in the state analytically, in order to reduce the variance and increase efficiency.

In [31], a fixed-lag smoother based on RBPF is proposed, focused on estimating the probability density function of the mode regulating the jump Markov system, $\theta[k]$. Then, the mode-conditioned estimates are computed by unscented Kalman smoother (UKS). Analogously, in [32] a new smoothing RBPF is proposed to track sharp maneuvers in a multiple-sensors network.

Another modification of particle filter smoothing is based on Markov chain Monte Carlo (MCMC) methods to generate the particles as an alternative to direct sampling. They use Markov chains to generate dependent samples from a certain probability density function (pdf) and therefore avoid the depletion problems of sequential particle filters. MCMC has been applied in [33] to smoothing, and by [34] to exploit, additionally, external knowledge through constrained filtering. In complex conditions, such as hard constraints and multiple targets, MCMC-PF was able to avoid loss of tracks due to wrong premature decisions. Given a number of particles, it alleviates the depletion problem allowing the use of a longer lags for smoothing, leading to improved track accuracy.

The examples in the literature show moderate improvement of MCMC-PF approaches, especially in situations with hard maneuvers, with the cost of computation loads. So, in [31] the computational load of the PF-UKS is shown to be more than one order of magnitude higher than that of an IMM. For this reason, in ATC domain with no hard constraints, clear flight models and singletrajectory reconstruction (mainly using ID codes for association), authors focused on IMM to have a good tradeoff between efficiency and performance, leaving the potential extension to MCMC-PF smoothers for future works.

In the proposed schema, each trajectory is divided in segments whose MoF is selected among a predefined set using a multiple hypothesis tracking technique over the whole trajectory. The reconstruction is finally done using an optimal forward-backward smoother composed of three extended Kalman filters, with the parameters of dynamic models adapted in each segment to the corresponding MoF. Meanwhile, in our approximation, a novel approach, to be named Model-Based Reconstruction (MBR), has been used: reconstruction is performed through variable structure IMM smoothers adapted to a previously estimated MoF sequence instead of directly applying a finite set of predefined MoFs. This permits to take advantage of the adaptability of variable structure IMM filters with respect to uncertainty in the maneuvering state, while exploiting the computed sequence of MoF, and therefore taking advantage of the regularity of maneuvers of commercial aircraft. The reconstruction process also exploits kinematic measurements when available (using ADS-B or Mode S Enhanced Surveillance ADD $[1,25]$ ).

The final output is a 3D reconstructed trajectory, including MoF and a smoothed curve. The problem is divided into two decoupled systems for horizontal $(\mathrm{XY})$ and vertical $(\mathrm{Z})$ coordinates, as usual in ATC domain. We will detail the horizontal smoothing procedure in this paper while the process in the vertical direction is only briefly summarized.

The paper contains eight sections. Section 2 describes the complete MBR structure. Sections 3-5 detail the reconstruction processes for trajectory segmentation, parameter estimation and smoothing for the horizontal coordinates. Section 6 presents the results of our approach and compares it with competing methods on simulated trajectories, Section 7 includes a brief description of the vertical dimension smoothing algorithms, and finally Section 8 summarizes our conclusions.

\section{System structure for model-based reconstruction}

In this section we put the reconstruction problem in context, introducing the preprocessing steps, and the general IMM smoothing approach (in Section 2.1). Then, in Section 2.2 we extend this classical approach to introduce our smoothing approach.

The sensors considered as data sources for this problem are: primary/secondary radars, wide-area multilateration (WAM) plots and navigation reports sent by aircraft (Automatic Dependent Surveillance, ADS). While primary radars (PSR) provide basic 2D information (range, azimuth), the secondary surveillance radars (SSR) provide 3D data (range, aximuth, barometric height), identification code (modes $\mathrm{A} / \mathrm{S}$ ), and the most recent Mode-S SSR provide extended reports with on-board velocity information (aircraftderived data, ADD).

Preprocessing includes coordinate transformation to the fusion coordinates of all data sources, data-to-trajectories association and bias estimation and correction. The data association organizes all data in the trajectories to be reconstructed, and also uses the identity information available in the ATC domain: ICAO SSR Mode-S code, time-position and velocity compatibility, etc., in order to simplify the problem and gain maximum reliability, since reconstruction algorithms are very sensitive to association or incorrect sensor error models.

Random error components are taken into account through noise covariance matrices, which are dependent on the sensor technology of each data. The required sensor modeling approach must be highly robust to avoid bad performance induced by real data problems. With respect to systematic errors, specific bias models are applied to each family of sensors mentioned above. More specific details about the complete SASS-C and OTR architecture and descriptions of association and bias-correction processes can be found in $[23,24,1]$. In this paper we assume bias has been corrected before the trajectory reconstruction, all data are correctly associated, and covariance of every measure has been calculated.

\subsection{IMM smoothing}

In general terms, the IMM algorithm [26-28] is suitable for applications with dynamic systems regulated with a predefined collection of operating modes. This is the case of maneuvering targets whose types of motion are in general members of a finite set of known alternatives. Then, a jump Markov linear system, where 
the state vector $x[k]$ can be modeled by a collection of linear models whose characteristics are regulated by the type of motion mode, $\theta[k] \in\left\{M_{1}, \ldots, M_{N}\right\}$, active during the time period $\left[t_{k}, t_{k+1}\right]$ can be defined:

$\mathbf{x}[k+1 \mid k]=\mathbf{F}(\theta[k], k) \mathbf{x}[k]+\mathbf{q}(\theta[k], k)$

$z[k]=H \mathbf{x}[k]+w[k]$

where $z[k]$ is the measurement, affected by a constant projection $H$ and additive noise $w[k]$, while the active dynamic model is regulated by transition matrix $F(\theta[k], k)$ and plant-noise process $q(\theta[k], k)$, both depending on the active motion mode at time instant $k, \theta[k]$. The mode-of-flight variable, $\theta[k]$, is modeled by means of first-order Markov chain whose transition probabilities are given by:

$\pi_{i j}=P\left(\theta_{k}=M_{j} \mid \theta_{k-1}=M_{i}\right)$

So, it is an extended estimation problem containing continuous and discrete state variables. With no knowledge apart from the sensor data, an optimum scheme would enumerate and average all hypothetical state sequences, $\theta^{K}=\theta[0], \ldots, \theta[k], \theta[i] \in\left\{M_{1}, \ldots\right.$ ,$\left.M_{N}\right\}, i \in\{0, \ldots, k\}$, weighted with the corresponding probabilities. In [3], an enumeration of hypotheses for sequences of $\theta^{K}$ is considered, based on MHT with pruning rules to reduce the number of evaluated sequences. For simplification, we will denote the plant noise process in (1), corresponding to mode $i$ as $q_{i}[k]$, $q_{i}[k] \equiv q\left(\theta[k]=M_{i}, k\right)$.

The IMM algorithm maintains the number of modes constant, and approximates the probability density function with a Gaussian mixture of $N$ modes, each centered at a matched Kalman filter defined with first and second order moments $\hat{x}^{j}[k], P^{j}[k]$, together with an estimation of the probability of the target being in each of them, $\mu^{i}[k]$. IMM has the advantage that it is quick to adapt to changes and close to the theoretically optimum solution [26].

In the reconstruction problem (also called smoothing or "retrodiction" [20]), the use of forward-backward filters has been proposed in applications such as fixed-lag smoothing, using forwardbackward alpha-beta filters [29], Kalman filters [18] and IMM filters $[19,20,30]$. In the last case, a fixed-lag smoothing method uses the basic IMM approach in a state-augmented system, with two IMM filters, propagating in the time forward and back-ward directions. The first problem with this IMM type is to find a set of models matched to the actual trajectory. This subject is far to be solved [22]. The second is to control, inside the IMM, the transitions among different model sets [27] (assign initial probabilities and obtain initial estimates and corresponding error covariance matrices). The formulation of IMM can be directly extended to the retrodiction problem (as proposed in [20]), with the probability density conditioned to all available observations and sequences of target states. Applying the total probability sum to the raw problem we would have the state density function conditioned to all measurements calculated as follows:

$$
\begin{aligned}
& p(x[k] \mid z[0], \ldots z[n]])= \\
& \left.\sum_{\theta[0], \ldots, \theta[n]} p(x[k], \theta[0], \ldots, \theta[n] \mid z[0], \ldots z[n]]\right)= \\
& \left.\sum_{\theta[0], \ldots, \theta[n]} p(x[k] \mid \theta[0], \ldots, \theta[n]) p(\theta[0], \ldots, \theta[n] \mid z[0], \ldots z[n]]\right)
\end{aligned}
$$

This sum may be unaffordable because the number of terms increases exponentially with the time length of sequence (all possible combinations of modes $\theta^{n}$ ). Again, the IMM approximation uses normal mixtures with fixed number of components:

$p(x[k] \mid z[0], \ldots z[n]]) \approx \sum_{j} \mu^{s . j}[k \mid n] \mathbf{N}\left(x[k] ; \hat{x}^{j}[k \mid n], P^{j}[k \mid n]\right)$

where $\mu^{s . j}[k \mid n]$ is the "smoothed" mode probability. Previous jumplinear models have been applied to smoothing or "retrodiction" problems. For instance, Helmick and Blair [18] used a smoothed mode probability, obtained after a forward-backward combination of modes:

$\mu^{s . j}[k \mid n]=P\{\theta[k]=M j \mid z[0], \ldots z[n]\}=\frac{1}{c} d^{j}[k] \mu^{j}[k]$

where $c$ is a normalization constant term, $\mu^{j}[k]$ is the forward probability mode, and $d^{j}[k]$ is the backward likelihood. This last term can be approximated as an $n$-dimensional Gaussian residual between forward filtered and backward predicted estimators, $\hat{x}^{f w d}[k \mid k]$ and ${ }^{b k d}[k \mid k+1]$, respectively with covariance matrices $P^{f w d}[k \mid k]$ and $P^{b k d}[k \mid k+1][18]:$

$d^{j}[k]=N_{n}(\bar{a} ; \overline{0}, C)$

$\bar{a}=\hat{x}^{b k d}[k \mid k+1]-\hat{x}^{f w d}[k \mid k]$

$C=P^{b k d}[k \mid k+1]+P^{f w d}[k \mid k]$

Being $N_{n}(\bar{x} ; \bar{c}, C)$ the Gaussian pdf evaluated at $\bar{x}$ with parameters $\bar{c}$ (mean) and $C$ (covariance). In Eq. (6) $\bar{c}=\overline{0}$ since it is assumed both estimates are unbiased, and $C$ is the sum of covariances assuming they are uncorrelated.

However, a classical smoothing approach based on IMM filters with fixed structure and parameters may show suboptimal behavior when none of the models achieves dominant probability, for instance, in situations where there are weak maneuvers and the structure switches from one model to another. This type of cases would not be solved by a classical maneuver detector [21] as commented in the introduction, since there are not significant deviations to identify the dominant mode.

This approach may show suboptimal performance in certain situations (i.e., in long segments with slow longitudinal accelerations that typically occur before landing). This limitation may have certain impact when the objective is to reconstruct trajectories of aircraft with different maneuvering capabilities. In these cases using a segmented input can have significant advantages over the use of averaged mode probabilities.

\subsection{Overall approach for model-based IMM smoothing}

The proposed process for horizontal trajectory reconstruction contains three main steps [24]:

- Trajectory segmentation into contiguous time intervals and identification of mode of flight (detailed in Section 3).

- Parameter estimation for each segment, and segment validation (Section 4).

- Model-based reconstruction with IMM smoothing (Section 5).

The IMM smoothing approach contains three modes, matched to the typical aircraft behavior in controlled airspace: uniform motion $\left(\mathrm{M}_{1}\right)$, coordinated turns $\left(\mathrm{M}_{2}\right)$ and longitudinal maneuvers with constant acceleration $\left(\mathrm{M}_{3}\right)$. The cinematic parameters and transition probabilities are tuned accordingly to the extracted mode probabilities, so that the structure is intended to reconstruct trajectories following a specific model with high accuracy, and at the same time generalizes to hybrid trajectories containing a composition of the simple patterns, exploiting the adaptability of IMM structures. Its parameters can be adjusted to achieve good trade-off of both behaviors (reconstruction accuracy in specific motion types and quick transitions).

The first process is based on segmenting data into homogeneous blocks corresponding to the Mode of Flight (MoF). Given the full sequence of time-ordered sensor measurements associated to the trajectory, $z[0], \ldots, z[n]$, the segmentation problem consists in finding an appropriate partition of measurements in intervals with the same MoF, $\wp=\left\{s_{i}\right\}$ : 
$\{z[0], \ldots, z[n]\}=\bigcup_{i=1}^{S} s_{i} ; \quad s_{i}=\left\{z\left[k_{i, \text { min }}\right], \ldots, z\left[k_{i, \max }\right]\right\}$

Each segment $s_{i}$ is described by its pair of minimum and maximum indexes $\left(k_{i, \min }, k_{i, \max }\right)$. This segmentation of measurements in blocks is equivalent to a partition of the trajectory into time subintervals $\tau_{i}$, where $t_{k}$ represents the detection time of measurement $z[k]$ :

$\wp \equiv \cup_{i=1}^{S} \tau_{i}=\left[t_{1, \min }, t_{2, \min }\right] \cup\left[t_{2, \min }, t_{3, \min }\right] \cup \cdots \cup\left[t_{S, \min }, t_{S, \max }\right]$

With the segmentation output in the first stage, a simple approach could try to exploit the MoF directly as an estimated sequence of modes, $\hat{\theta}[k]=\operatorname{MoF}\left(s_{i}\left(t_{k}\right)\right)$, and apply a single-mode Kalman filter for each segment. This approach would be similar to the one proposed in [3]. However, the segmentation may be subject to potential errors and model mismatches, with the evident risk of translating segmentation errors to reconstruction.

To avoid this problem, our proposed solution divides the later processing in two steps. As described in Section 4, it first tries to perform a segment parameter estimation procedure calculating magnitudes such as accelerations or turn radius for different MoF segments, and then validates the parameters and segmentation using a residual analysis. Finally, as detailed in Section 5, it keeps an IMM algorithm conditioned to the sequence of extracted MoF segments, to maintain high accuracy in segment borders. In this final IMM smoothing system, the transition parameters regulating the Markov chain are adjusted depending on the presence of MoF transitions:

$\pi_{i j}[k]=\pi_{i j}\left(t_{k}\right)=f(\wp)=f\left(\operatorname{MoF}\left(\left\{s\left(t_{k-1}\right), s\left(t_{k}\right), s\left(t_{k+1}\right)\right\}\right)\right)$

So, the transition probability at time $\mathrm{k}$ depends on the context and presence of close transitions of MoF.

\section{Mode-of-flight identification}

The segmentation is the first step in the proposed model-based reconstruction method. Searching a global optimum for segmentation would mean the enumeration of all potential partitions and their evaluation with fitting metrics, which is computationally unaffordable. The most general approaches for segmenting time series are summarized in [35], and extended in [36]. However, for large amounts of raw data, which is the case here, general algorithms may not be affordable. Authors have previously formulated the segmentation problem and explored data mining techniques to improve the results [37].

We defined a segmentation process based on mode identification with an IMM carried out before reconstruction. Besides, if Aircraft Derived Data (ADD) information is available, a complementary segmentation method was designed. Both segmentations can be combined.

The IMM algorithm for MoF identification is based on three dynamic models, whose function is to estimate the probability of the target developing significant transversal and longitudinal deviations with the goal of finding the transitions or edges between segments. The maneuvering modes are aimed at detecting maneuvers aligned with their oriented plant covariance matrices, so their probabilities correspond to the presence of transitions among the different modes of flight. The general MoF identification scheme is depicted in Fig. 1.

The three modes have a common 2D prediction equation at constant velocity but with different uncertainty model, the differences are in the plant noise processes associated to each model:

$$
\left[\begin{array}{c}
\hat{x}_{i}[k+1 \mid k] \\
\hat{v}_{x_{i}}[k+1 \mid k] \\
\hat{y}_{i}[k+1 \mid k] \\
\hat{v}_{y_{i}}[k+1 \mid k]
\end{array}\right]=\left[\begin{array}{cccc}
1 & \Delta t & 0 & 0 \\
0 & 1 & 0 & 0 \\
0 & 0 & 1 & \Delta t \\
0 & 0 & 0 & 1
\end{array}\right]\left[\begin{array}{c}
\hat{x}_{i}[k \mid k] \\
\hat{v}_{x_{i}}[k \mid k] \\
\hat{y}_{i}[k \mid k] \\
\hat{v}_{y_{i}}[k \mid k]
\end{array}\right]+q_{i}[k]
$$

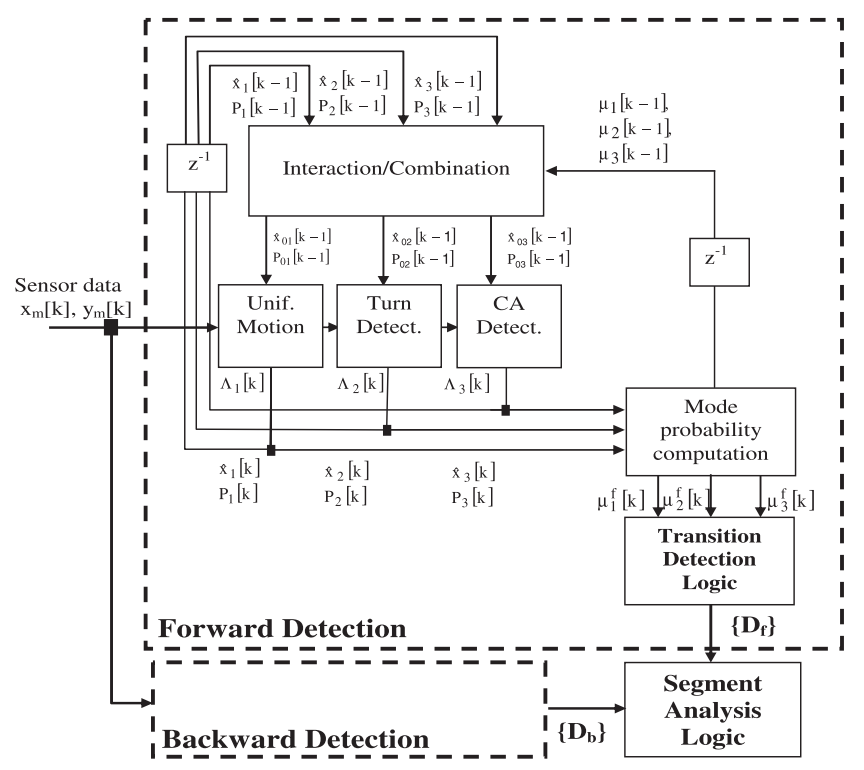

Fig. 1. IMM structure for MoF identification.

being $(x, y)$ and $\left(v_{x}, v_{y}\right)$ the position and velocity in stereographic plane, $\Delta t$ the time elapsed since last update $\left(\Delta t=t_{k+1}-t_{k}\right)$ and $q_{i}[k]$ the plant noise process of $i$ th IMM mode (Eq. (1)). The uniform mode $\left(\mathrm{M}_{1}\right)$ assumes no uncertainty in the prediction (suitable for constant velocity flight segments typical in controlled traffic), so covariance is zero $\left(Q_{1}[k]=0\right)$, while the two maneuvering models, $\mathrm{M}_{2}, \mathrm{M}_{3}$, have noise processes defining "oriented" covariance matri-ces, longitudinally (for $\mathrm{M}_{2}$ ) and transversally (for $\mathrm{M}_{3}$ ) along the potential aircraft maneuvering directions (as illustrated in [37]). These modes allow deviations along the manoeuver directions, and so would take higher probabilities as soon as the other modes do not allow deviations from the predictions.

Besides the oriented term, the plant noise processes contain a homogeneous term to achieve higher stability with irregular maneuvers, accounting model deviations in unknown directions. So, the final covariance matrix for $i$ th mode, $Q_{i}[k]$, is the sum of both, where the directional part depends on the aircraft estimated heading, , while the homogeneous term consider other potential effects leading to model mismatch. The parameters with relative strengths of these terms, $\sigma_{g a}^{2}, \sigma_{d a}^{2}$ are tuned as a trade-off between detection sensitivity in transitions and mode stability in a steady situation:

$$
\begin{gathered}
Q_{i}(\Delta t)=\sigma_{g a}^{2}\left[\begin{array}{cccc}
\Delta t^{4} / 4 & \Delta t^{3} / 2 & 0 & 0 \\
\Delta t^{3} / 2 & \Delta t^{2} & 0 & 0 \\
0 & 0 & \Delta t^{4} / 4 & \Delta t^{3} / 2 \\
0 & 0 & \Delta t^{3} / 2 & \Delta t^{2}
\end{array}\right]+ \\
\sigma_{d a}^{2}\left[\begin{array}{cccc}
c_{i}^{2} \Delta t^{4} / 4 & c_{i}^{2} \Delta t^{3} / 2 & c_{i} s_{i} \Delta t^{4} / 4 & c_{i} s_{i} \Delta t^{3} / 2 \\
c_{i}^{2} \Delta t^{3} / 2 & c_{i}^{2} \Delta t^{2} & c_{i} s_{i} \Delta t^{3} / 2 & c_{i} s_{i} \Delta t^{2} \\
c_{i} s_{i} \Delta t^{4} / 4 & c_{i} s_{i} \Delta t^{3} / 2 & s_{i}^{2} \Delta t^{4} / 4 & s_{i}^{2} \Delta t^{3} / 2 \\
c_{i} s_{i} \Delta t^{3} / 2 & c_{i} s_{i} \Delta t^{2} & s_{i}^{2} \Delta t^{3} / 2 & s_{i}^{2} \Delta t^{2}
\end{array}\right]
\end{gathered}
$$

Eq. (11) is the plant noise covariance, where $c_{i}, s_{i} ; i=\{2,3\}$ are the trigonometric functions projecting the uncertainty along the direction of motion. The corresponding values for mode $\mathrm{M}_{2}$ (transversal deviation) aligned to transversal direction across to aircraft orientation, $\phi+\pi / 2$, and mode $\mathrm{M}_{3}$ (longitudinal deviation), aligned to the aircraft orientation $\phi$ are: 


$$
\begin{aligned}
& c_{2}=\frac{\hat{v}_{y}}{\sqrt{\hat{v}_{x}^{2}+\hat{v}_{y}^{2}}} ; S_{2}=\frac{-\hat{v}_{x}}{\sqrt{\hat{v}_{x}^{2}+\hat{v}_{y}^{2}}} \\
& c_{3}=\frac{\hat{v}_{x}}{\sqrt{\hat{v}_{x}^{2}+\hat{v}_{y}^{2}}} ; \quad s_{3}=\frac{\hat{v}_{y}}{\sqrt{\hat{v}_{x}^{2}+\hat{v}_{y}^{2}}}
\end{aligned}
$$

It is interesting to notice that they are non-linear models since the plant noise process depends on the estimate vector (velocity). Therefore, the Kalman assumptions of Gaussian-linear systems are not fully applicable and we would have suboptimal estimate for these modes. However the approximation can be quite reasonable in the situations of interest: commercial air traffic in en-route and terminal areas, where the magnitude of speed is enough to have a quite reasonable estimation of orientation and lead to stable covariance matrix.

After the mode probabilities are computed, the method to determine the MoF segments begins with an edge detection process to locate the beginnings and ends of typical maneuvers. This is done by analyzing the drops in uniform mode probability to identify the possible edges of segments:

$$
\begin{aligned}
& D_{f}=\left\{k\left|\mu_{U}^{f}[k]<T h_{D} \wedge \mu_{U}^{f}[k-i] \geqslant T h_{D} ; \forall i\right| t_{k}-t_{k-i}<t_{W D}\right\} \\
& D_{b}=\left\{k\left|\mu_{U}^{b}[k]<T h_{D} \wedge \mu_{U}^{b}[k+i] \geqslant T h_{D} ; \forall i\right| t_{k+i}-t_{k}<t_{W D}\right\}
\end{aligned}
$$

$D_{f}, D_{b}$ being the resulting sets of indexes corresponding to potential maneuver detections in the forward and backward filters, respectively. A sample index $k$ (with corresponding detection time $t_{k}$ ) is added to the set when the probability of uniform mode $\left(M_{1}\right)$, $\mu_{U}[k]$ (forward and backward, as denoted by the super-index), drops below a threshold $T h_{D}$, and the samples before the drop (respectively, after) are above this threshold at least for a minimum time window, $t_{W D}$. As pointed out in [37], both sets have complementary characteristics: forward-run transitions from uniform motion to maneuvers are typically quick (high residual makes the zero-noise uniform mode lose all probability), whereas transitions from maneuvers returning back to uniform state are slow (the end of maneuver usually needs more samples to impact likelihoods and mode probabilities). Due to the symmetry in time, edges in probability from both runs can be complementarily used to identify the segments.

After edge detection, the probabilities for the two maneuvering modes are considered, $\mu_{j}^{f w d}[k], \mu_{j}^{b k d}[k]$, with $j \in\left\{M_{2}, M_{2}\right\}$. The condition for a valid maneuvering mode is that it must contain at least $50 \%$ of averaged mode probability along the tested segmented interval, together with a validation test based on normalized residual to be detailed later. If a segment does not pass the test, it is labeled as "unknown". As explained later, the reconstruction applies a robust (but not so accurate) default process with "unknown" segments.

After segment detection, a preliminary division of the sequence into MoF segments is available. This will probably lead to a certain number of false divisions due to noise. This problem is dealt through a refinement in a second phase over initial segmentation. This phase tries to merge and reclassify short segments with adjacent segments both for uniform and maneuvering segments. The metric for this process is the normalized mode residual to be described in Section 4, so that the best decision is taken to minimize the residual of the resulting segments. Fig. 2 shows an example where a very short interval is discarded and attached to an adjacent segment. Then, a series of three adjacent uniform segments (black line) are reconnected into one.

We have explained the segmentation process based on forward-backward IMM probabilities. An analogous process is carried out when ADD velocities are available, considering the estimated longitudinal and transversal accelerations based on cinematic measurements.

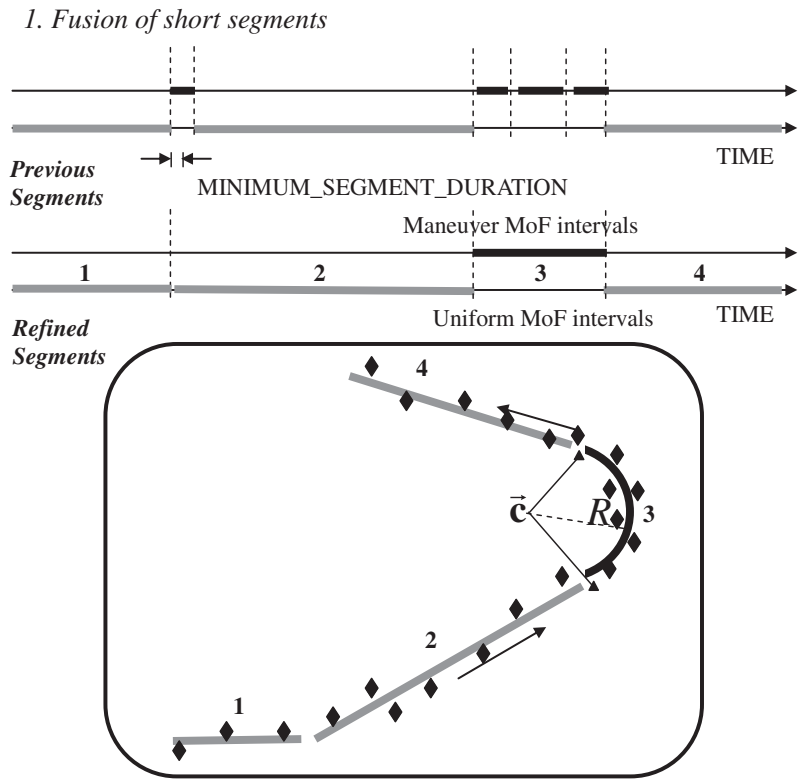

Fig. 2. Illustration of rules for MoF segmentation.

\section{Parameter estimation and segment validation}

A weighted least squares (WLS) estimates the parameters corresponding to each MoF segment $s_{i}$, containing the observations $\left\{z\left[k_{i, \min }\right], \ldots, z\left[k_{i, \max }\right]\right\}$. The estimation provides the segment parameters, $\left\langle\bar{P}\left(s_{i}\right)\right\rangle$, which are also used to validate these segments as precondition to their use in reconstruction (otherwise, the default mode corresponding to "unknown" mode will be applied) [24].

The estimation is specific for each one of the three models considered in the reconstruction. In the case of constant-acceleration motion (CA), the parabolic motion model in Cartesian coordinates is applied, with six parameters (positions, velocities, and accelerations on the stereographic plane at initial segment time). The relation between each measure and the parameters for modeling the segment is given by:

$$
\begin{aligned}
{\left[\begin{array}{l}
x_{m}[k] \\
y_{m}[k]
\end{array}\right]=} & H\left(t_{k}\right) P^{-}+n^{-}[k]=\left[\begin{array}{cccccc}
1 & t_{k} & \frac{t_{k}^{2}}{2} & 0 & 0 & 0 \\
0 & 0 & 0 & 1 & t_{k} & \frac{t_{k}^{2}}{2}
\end{array}\right]\left[\begin{array}{c}
x_{0} \\
v x_{0} \\
a x_{0} \\
y_{0} \\
v y_{0} \\
a y_{0}
\end{array}\right] \\
& +\left[\begin{array}{c}
n_{x}[k] \\
n_{y}[k]
\end{array}\right]
\end{aligned}
$$

$\left(x_{m}[k], y_{m}[k]\right)$ are observations expressed in Cartesian plane, for each time $k ; t_{k}$ is the time elapsed between $k$ th measurement and starting time; $\left(x_{0}, y_{0}\right),\left(v_{x 0}, v_{y 0}\right)$ and $\left(a_{x 0}, a_{y 0}\right)$ are the estimated initial values for position, velocity, acceleration of segment, respectively. $\vec{n}[k]$ is the observation noise, with associated covariance matrix $\mathbf{R}_{k}$. The WLS segment parameters are given by:

$$
\begin{aligned}
& \langle\bar{P}\rangle=\left\lfloor\begin{array}{llllll}
\left\langle x_{0}\right\rangle & \left\langle v_{x_{0}}\right\rangle & \left\langle a_{x_{0}}\right\rangle & \left\langle y_{0}\right\rangle & \left\langle v_{y_{0}}\right\rangle & \left\langle a_{y_{0}}\right\rangle
\end{array}\right\rfloor^{t} \\
& \left.=\sum_{k} H\left(t_{k}\right)^{T} R_{k}^{-1} H\left(t_{k}\right)\right)^{-1} \sum_{k} H\left(t_{k}\right)^{T} R_{k}^{-1} \vec{x}_{m}[k]
\end{aligned}
$$

It is known [38] that this WLS is, under Gaussianity and independence conditions, the best linear unbiased estimator (BLUE) if each weight is equal to the inverse of the variance of the measurement, $\mathbf{R}_{k}$. "Best" means it has minimum variance. 
Analogously, the average parameters of a uniform segment are computed assuming constant velocity, $\langle\bar{P}\rangle=\left[\begin{array}{lll}\left\langle x_{0}\right\rangle & \left\langle v_{x_{0}}\right\rangle & \left\langle y_{0}\right.\end{array}\right.$ $\left.\left\langle v_{y_{0}}\right\rangle\right]^{t}$, with the same equations over a reduced matrix $H$. The uniform segment parameters are used to compute residual and validate the segments classified with this category (the most usual in commercial flights moving along airways).

The problem with the turn maneuver model is that linear regression is not possible because the model contains non-linear equations. Instead, a linear regression is performed in a transformed space with linear relations. If all measures would belong to a common circle, we would have the relation:

$x_{m}^{2}[k]+y_{m}{ }^{2}[k]+a x_{m}[k]+b y_{m}[k]+c=0$

These equations can be re-arranged in matrix form to:

$$
\left[\begin{array}{ccc}
x_{m}[1] & y_{m}[1] & 1 \\
x_{m}[2] & y_{m}[2] & 1 \\
\ldots & \ldots & \ldots \\
x_{m}[N] & y_{m}[N] & 1
\end{array}\right]\left[\begin{array}{l}
a \\
b \\
c
\end{array}\right]=\left[\begin{array}{c}
-\left(x_{m}^{2}[1]+y_{m}^{2}[1]\right) \\
-\left(x_{m}^{2}[2]+y_{m}^{2}[2]\right) \\
\ldots \\
-\left(x_{m}{ }^{2}[N]+y_{m}{ }^{2}[N]\right)
\end{array}\right]
$$

and renaming matrices as:

$X\left[\begin{array}{lll}a & b & c\end{array}\right]^{t}=\vec{Y}$

Finally the parameters can be estimated with WLS method:

$\left[\begin{array}{lll}\langle a\rangle & \langle b\rangle & \langle c\rangle\end{array}\right]^{t}=\left(X^{T} X\right)^{-1} X^{T} \vec{Y}$

Finally, these auxiliary estimates are used to compute the interpolating circle parameters (center $\widehat{C}$ and radius $\widehat{R}$ ):

$\langle C\rangle=\left[\begin{array}{l}\left\langle c_{X}\right\rangle \\ \left\langle c_{Y}\right\rangle\end{array}\right]=\left[\begin{array}{l}-\langle a\rangle / 2 \\ -\langle b\rangle / 2\end{array}\right] ; \quad\langle R\rangle=\sqrt{-\langle c\rangle+\frac{\langle a\rangle^{2}}{4}+\frac{\langle b\rangle^{2}}{4}}$

Finally, to increase the reconstruction robustness, a validation is applied to all segments with a chi-squared test, over the accumulated residuals of measurements with respect to their assumed motion model. This validation avoids the undesirable effects of using wrong parameters for reconstruction. The "ideal" trajectory segment, calculated from the estimated parameters, is compared with measurements in the interval to obtain the averaged normalized residual. If the averaged residual exceeds a threshold, the segment is classified as "unknown". The normalized residual of a segment defined between indexes $\left[k_{1}, k_{2}\right]$, considering the degrees of freedom of probability density, is computed as:

$r_{k}=\left[\begin{array}{l}\langle x[k]\rangle-x_{m}[k] \\ \langle y[k]\rangle-y_{m}[k]\end{array}\right]$

$e_{s}=\frac{1}{2\left(k_{2}-k_{1}+1\right)-P} \sum_{k=k_{1}}^{k_{2}} r_{k}^{t} R_{k}^{-1} r_{k}$

where $(\langle x[k]\rangle,\langle y[k]\rangle)$ is the WLS or regression based prediction accordingly to segment type (the parameters corresponding to uniform, turning or accelerated segment), and $P$ is a value to be defined later for each model. This proposed statistic for validation, $e_{s}$, follows a normalized chi-squared pdf, $\frac{1}{n} \chi_{n}^{2}$, with " $n$ " the number of degrees of freedom of distribution (under null hypotheses of correct model, it has mean value of 1 and variance of $1 / n$ ) if segment interpolation errors are neglected in relation to measurement errors.

This value $n$, is given by the number of measurements contained in the block multiplied by two (since we have 2D observations), minus the dimension of the segments parameters vector: $(P=4$ in uniform segment, $P=6$ in CA segment, $P=3$ in turn segment). So, $n=2\left(k_{2}-k_{1}+1\right)-P$.

The threshold used to validate the normalized residual depends on the number of measurements in the interval. Table 1 shows the thresholds designed for a maximum of $0.1 \%$ probability of rejecting a correct segment due to the measurement noise.
Table 1

Threshold values for validating MoF segments.

$\begin{array}{lllllll}\text { DF: } \mathrm{n} & 10 & 50 & 100 & 150 & 200 & 250 \\ \mathrm{Th}(0.1 \%) & 2.96 & 1.73 & 1.49 & 1.4 & 1.34 & 1.3\end{array}$

\section{Model based IMM smoothing}

The IMM smoothing filter for ATC reconstruction contains 3 Kalman filters, matched to the typical aircraft behavior in controlled airspace: uniform motion $\left(\mathrm{M}_{1}\right)$, coordinated turns $\left(\mathrm{M}_{2}\right)$ and longitudinal manoeuvres with constant acceleration $\left(\mathrm{M}_{3}\right)$. The three modes used in the IMM have a $4 \mathrm{D}$ state vector, with $2 \mathrm{D}$ position and velocity in the 2D stereographic plane, $\bar{x}[k]=\left[\hat{x}[k] \hat{v}_{x}[k]\right.$ $\left.\hat{y}[k] \hat{v}_{y}[k]\right]^{t}$, and their associated $4 \times 4$ error covariance matrices, $P[k]$. They have different state prediction models, with specific control inputs regulating their dynamics (depending on parameters extracted from maneuvering segments) and also differences in their associated plant noises.

The algorithm is based on the segmented sequence $s_{i}, i=1, \ldots, S$, with associated MoF, time interval and segment motion parameters, estimated using the WLS estimators as detailed in Section 4: $\operatorname{MoF}\left(s_{i}\right)$, $\tau_{i}\left(s_{i}\right),\left\langle P\left(s_{i}\right)\right\rangle$. The general idea was sketched in [24], the modes in IMM are affected by the prediction parameters estimated with WLS.

The mode $\mathrm{M}_{1}$, optimum for uniform segments, uses a noise-free constant-velocity motion model for state prediction:

$\left[\begin{array}{l}\hat{x}[k+1 \mid k] \\ \hat{v}_{x}[k+1 \mid k] \\ \hat{y}[k+1 \mid k] \\ \hat{v}_{y}[k+1 \mid k]\end{array}\right]=\left[\begin{array}{cccc}1 & \Delta t & 0 & 0 \\ 0 & 1 & 0 & 0 \\ 0 & 0 & 1 & \Delta t \\ 0 & 0 & 0 & 1\end{array}\right]\left[\begin{array}{c}\hat{x}[k \mid k] \\ \hat{v}_{x}[k \mid k] \\ \hat{y}[k \mid k] \\ \hat{v}_{y}[k \mid k]\end{array}\right]$

For $\mathrm{M}_{3}$ (longitudinal acceleration), the prediction equations for the CA segment are given by:

$$
\left[\begin{array}{c}
\hat{x}[k+1 \mid k] \\
\hat{v}_{x}[k+1 \mid k] \\
\hat{y}[k+1 \mid k] \\
\hat{v}_{y}[k+1 \mid k]
\end{array}\right]=\left[\begin{array}{cccc}
1 & \Delta t & 0 & 0 \\
0 & 1 & 0 & 0 \\
0 & 0 & 1 & \Delta t \\
0 & 0 & 0 & 1
\end{array}\right]\left[\begin{array}{c}
\hat{x}[k \mid k] \\
\hat{v}_{x}[k \mid k] \\
\hat{y}[k \mid k] \\
\hat{v}_{y}[k \mid k]
\end{array}\right]+\frac{1}{2} \Delta t\left[\begin{array}{c}
\Delta t\left\langle a_{x s}\right\rangle \\
2\left\langle a_{x s}\right\rangle \\
\Delta t\left\langle a_{y s}\right\rangle \\
2\left\langle a_{y s}\right\rangle
\end{array}\right]+\bar{q}_{C A}[k]
$$

In this case, the control input vector $\left(\left\langle a_{x s}\right\rangle,\left\langle a_{y s}\right\rangle\right)$ is the longitudinal acceleration estimated with the WLS, and $\bar{q}_{C A}[k]$ is the plant noise process.

With respect to $\mathrm{M}_{2}$ (turn model) the prediction model assumes the aircraft is moving at constant speed in a coordinated turn, and so target trajectory describes a circular arc whose parameters (circle centre $\left(\left\langle C_{x}\right\rangle,\left\langle C_{y}\right\rangle\right)$, and radius $\left.\langle R\rangle\right)$ estimated as explained above. This type of dynamic is described by non-linear equations. If the variation in orientation between consecutive updates is low enough (which is reasonable in civil air traffic), these equations of circular dynamics can be linearized with a first order approximation [39]. Mode $\mathrm{M}_{2}$ uses an extended Kalman filter with equations:

$\left[\begin{array}{c}\hat{x}[k+1 \mid k] \\ \hat{v}_{x}[k+1 \mid k] \\ \hat{y}[k+1 \mid k] \\ \hat{v}_{y}[k+1 \mid k]\end{array}\right]=\left[\begin{array}{cccc}1 & \sin \left(\left\langle w_{s}\right\rangle \Delta t\right) /\left\langle w_{s}\right\rangle & 0 & -\left(1-\cos \left(\left\langle w_{s}\right\rangle \Delta t\right)\right) /\left\langle w_{s}\right\rangle \\ 0 & \cos \left(\left\langle w_{s}\right\rangle \Delta t\right) & 0 & -\sin \left(\left\langle w_{s}\right\rangle \Delta t\right) \\ 0 & \left(1-\cos \left(\left\langle w_{s}\right\rangle \Delta t\right)\right) /\left\langle w_{s}\right\rangle & 1 & \sin \left(\left\langle w_{s}\right\rangle \Delta t\right) /\left\langle w_{s}\right\rangle \\ 0 & \sin \left(\left\langle w_{s}\right\rangle \Delta t\right) & 0 & \cos \left(\left\langle w_{s}\right\rangle \Delta t\right)\end{array}\right]\left[\begin{array}{c}\hat{x}[k] \\ \hat{v}_{x}[k] \\ \hat{y}[k] \\ \hat{v}_{y}[k]\end{array}\right]$

where the "average" angular velocity of segment, $\left\langle w_{s}\right\rangle$, is derived from circle parameters and average aircraft speed (over the set of $L_{s}$ measures contained in segment $s$ ):

$\left\langle w_{s}\right\rangle=\frac{\sqrt{\bar{v}_{x}^{2}[k]+\bar{v}_{y}^{2}[k]}}{\langle R\rangle} ; \quad\left(\bar{v}_{x}, \bar{v}_{y}\right)=\frac{1}{L_{s}} \sum_{j}\left(\hat{v}_{x}[j], \hat{v}_{y}[j]\right)$ 
The plant noise processes of the two maneuvering models, $\mathrm{M}_{2}$, $\mathrm{M}_{3}$, have "oriented" covariance matrices, as the components used for MoF detection. They are used only in validated segments (modes $\left.M_{1}, M_{2}, M_{3}\right)$. If the segment is not validated $\left(M_{0}\right)$, the prediction equation removes the external parameters and uses an increased and spatially homogeneous (circular) plant-noise term in prediction, defining a plant noise covariance $(Q)$ with parameter $\sigma_{\text {Unk }}$ :

$$
\begin{gathered}
{\left[\begin{array}{c}
\hat{x}[k+1 \mid k] \\
\hat{v}_{x}[k+1 \mid k] \\
\hat{y}[k+1 \mid k] \\
\hat{v}_{y}[k+1 \mid k]
\end{array}\right]=\left[\begin{array}{cccc}
1 & \Delta t & 0 & 0 \\
0 & 1 & 0 & 0 \\
0 & 0 & 1 & \Delta t \\
0 & 0 & 0 & 1
\end{array}\right]\left[\begin{array}{c}
\hat{x}[k \mid k] \\
\hat{v}_{x}[k \mid k] \\
\hat{y}[k \mid k] \\
\hat{v}_{y}[k \mid k]
\end{array}\right]} \\
Q_{\text {Unk }}(\Delta t)=\sigma_{\text {Unk }}^{2}\left[\begin{array}{cccc}
\Delta t^{4} / 4 & \Delta t^{3} / 2 & 0 & 0 \\
\Delta t^{3} / 2 & \Delta t^{2} & 0 & 0 \\
0 & 0 & \Delta t^{4} / 4 & \Delta t^{3} / 2 \\
0 & 0 & \Delta t^{3} / 2 & \Delta t^{2}
\end{array}\right]
\end{gathered}
$$

The available ADD velocity data contained in the observations received from Mode-S and ADS reports are also used in the reconstruction process. It is reported as groundspeed and heading, which are first transformed, with their associated covariance matrix, to the stereographic frame. Then, these observations are integrated with the rest in the reconstruction filters by means of appropriate projection matrices. The projection matrices for 2D position $\left(H_{\text {pos }}\right), 4 \mathrm{D}$ position + velocity $\left(H_{\text {posvel }}\right.$, assuming the measure has in its first two rows the projected position and in the last rows the projected velocity) and $2 \mathrm{D}$ velocity $\left(H_{v e l}\right)$ are, respectively:

$$
\begin{aligned}
& H_{p o s}=\left[\begin{array}{llll}
1 & 0 & 0 & 0 \\
0 & 0 & 1 & 0
\end{array}\right] ; \quad H_{\text {posvel }}=\left[\begin{array}{cccc}
1 & 0 & 0 & 0 \\
0 & 0 & 1 & 0 \\
0 & 1 & 0 & 0 \\
0 & 0 & 0 & 1
\end{array}\right] \\
& H_{\text {vel }}=\left[\begin{array}{llll}
0 & 1 & 0 & 0 \\
0 & 0 & 0 & 1
\end{array}\right]
\end{aligned}
$$

Finally, the transition probability matrix, $T[k]=\pi_{i j}[k]$, is computed as:

$$
T[k]=\left[\begin{array}{ccc}
1-p_{U T}[k]-p_{U L}[k] & p_{U T}[k] & p_{U L}[k] \\
p_{T U}[k] & 1-p_{T U}[k] & 0 \\
p_{L U}[k] & 0 & 1-p_{L U}[k]
\end{array}\right]
$$

In (28) $p_{U T}, p_{U L}$ are, respectively, the probabilities of starting a transversal and a longitudinal maneuver from the uniform state (Uniform to Transversal, Uniform to Longitudinal), whereas, conversely, $p_{T U}$, $p_{L U}$ are the probabilities of doing transitions from maneuvers at transversal and longitudinal directions, respectively, to go back to uniform state. The adjustment of all IMM probability parameters is a process of finding the values leading to the best results. This is a non-trivial design, which can have an important impact on global performance, as authors have previously shown with the application of optimization techniques for real time trackers [40,41].

So, the MBR IMM smoother uses different models and parameters, conditioned to the presence and validation of different motion modes (the absence of a validated mode triggers a default model). The transition parameters are adjusted depending on the sequence of MoF segments. So, the four probabilities in (27) are not constant, but they are adapted depending on the presence of close transitions in the segment sequence $\left\{s_{i}\right\}$, and also considering the type of transition. We make them variable with the segmentation chain:

$\left\{\pi_{i j}[k]\right\}=f(\wp)=f\left(\operatorname{MoF}\left(\left\{s\left(t_{k-1}\right), s\left(t_{k}\right), s\left(t_{k+1}\right)\right\}\right)\right)$

The transition parameters are adapted according to the following rules:
//MODE UNIFORM AND NOT CLOSE TRANSITION

$$
\text { if } \begin{aligned}
s\left(t_{k}\right)=M_{1} \wedge & \left(t_{k+1}-t_{k} \geqslant t_{W T} \wedge t_{k}-t_{k-1} \geqslant t_{W T}\right) \\
& p_{U T}[k]=p U M_{\text {STABLE }} \\
& p_{U L}[k]=p U M_{\text {STABLE }} \\
& p_{T U}[k]=p M U_{\text {STABLE }} \\
& p_{L U}[k]=p M U_{\text {STABLE }}
\end{aligned}
$$

//MODE UNIFORM AND CLOSE TRANSITION

$$
\text { if } \begin{aligned}
s\left(t_{k}\right)=M_{1} \wedge & \left(t_{k+1}-t_{k}<t_{W T} \vee t_{k}-t_{k-1}<t_{W T}\right) \\
& p_{U T}[k]=p U M_{\text {TRANSITION }} \\
& p_{U L}[k]=p U M_{\text {TRANSITION }} \\
& p_{T U}[k]=p M U_{\text {TRANSITION }} \\
& p_{L U}[k]=p M U_{\text {TRANSITION }}
\end{aligned}
$$

\section{//MODE TURN AND NOT CLOSE TRANSITION}

$$
\text { if } \begin{aligned}
s\left(t_{k}\right)=M_{2} \wedge & \left(t_{k+1}-t_{k} \geqslant t_{W T} \wedge t_{k}-t_{k-1} \geqslant t_{W T}\right) \\
& p_{U T}[k]=p U M_{M A N} \\
& p_{U L}[k]=0 \\
& p_{T U}[k]=p T U_{M A N} \\
& p_{L U}[k]=p L U_{M A N}
\end{aligned}
$$

//MODE TURN AND CLOSE TRANSITION

$$
\text { if } \begin{aligned}
s\left(t_{k}\right)=M_{2} \wedge & \left(t_{k+1}-t_{k}<t_{W T} \vee t_{k}-t_{k-1}<t_{W T}\right) \\
& p_{U T}[k]=p U M_{\text {TRANSITION }} \\
& p_{U L}[k]=0 \\
& p_{T U}[k]=p M U_{\text {TRANSITION }} \\
& p_{L U}[k]=p M U_{\text {TRANSITION }}
\end{aligned}
$$

\section{//MODE CA AND NOT CLOSE TRANSITION}

$$
\text { if } \begin{aligned}
s\left(t_{k}\right)=M_{3} \wedge & \left(t_{k+1}-t_{k} \geqslant t_{W T} \wedge t_{k}-t_{k-1} \geqslant t_{W T}\right) \\
& p_{U T}[k]=0 \\
& p_{U L}[k]=p U M_{M A N} \\
& p_{T U}[k]=p T U_{M A N} \\
& p_{L U}[k]=p L U_{M A N}
\end{aligned}
$$

\section{//MODE CA AND CLOSE TRANSITION}

$$
\text { if } \begin{aligned}
s\left(t_{k}\right)=M_{3} \wedge\left(t_{k+1}-t_{k}<t_{W T} \vee t_{k}-t_{k-1}<t_{W T}\right) \\
p_{U T}[k]=0 \\
p_{U L}[k]=p U M_{\text {TRANSITION }} \\
p_{\text {TU }}[k]=p M U_{\text {TRANSITION }} \\
p_{L U}[k]=p M U_{\text {TRANSITION }}
\end{aligned}
$$

The specific IMM transition parameters used in this solution are characterized distinguishing stable motion and transitions: probability of transition from Uniform to Maneuvering modes (UM) when the state is stable uniform motion (pUM_STABLE, pMU_STABLE), stable maneuvering motion (both during turns or longitudinal accelerations) (pUT_MAN, pUL_MAN, pTU_MAN, pLU_MAN), and values for the transitions with both types of maneuvers (pUM_TRANSITION, pMU_TRANSITION). The separation between "stable" and "transition" is also controlled by a time window parameter, $t_{W T}$, considering the separation of current time with the previous or following transition. Besides these parameters of transition probabilities, plant noise variances of IMM reconstruction modes are increased during transitions to minimize potential overshoots.

Therefore, both mode parameters, $M_{j}[k]$, and transition probabilities, are conditioned to the transitions having occurred before and after the current time $t_{k}$, information directly extracted from the series of MoF values in the previous phase. As a particular case, the parameters are also adjusted for the transitions between validated MoF intervals and non-validated (or "unknown") motion segments. During an unknown motion mode, the IMM operates 
with different parameters, increasing the chance of moving from uniform to transversal and longitudinal deviations:

$$
\begin{aligned}
/ / \text { MODE UNKNOWN } & \\
\operatorname{ifs}\left(t_{k}\right)=M_{0} & \\
p_{U T}[k] & =p U M_{\text {UNKNOWN }} \\
p_{U L}[k] & =p U M_{\text {UNKNOWN }} \\
p_{T U}[k] & =p M U_{U N K N O W N} \\
p_{L U}[k] & =p M U_{U N K N O W N}
\end{aligned}
$$

The parameterization of IMM is a complex design process carried out with intensive analysis and evaluation in test scenarios. The most critical are covariances of plant-noise processes and transition probabilities, which must be jointly tuned to drive the final performance (see [40]). In this case, the information available after segmentation opens the possibility of a much more detailed parameterization considering the specific situation for reconstruction (stable motion, transition, unknown type, etc.). The values selected for the parameters described above were fixed after evaluation in simulated and real scenarios, although an exhaustive analysis of this topic is out of the scope of this paper.

As the final step for smoothing, the final reconstructed samples are computed with a weighted combination of forward-backward vectors from runs of the IMM structure in both directions (if available), with their inverse covariance matrices [18]. Denoting the filtered forward vector as $\hat{x}_{f}[k \mid 1: k]$, the one-step predicted backward vector (predicted to avoid combination of correlated estimators) as $\hat{x}_{b}[k \mid k+1: n]$, and their respective covariance matrices as $P_{f w d}[k \mid 1: k], P_{b k d}[k \mid k+1: n]$, the smoothed reconstruction sample $\hat{x}_{s}[k \mid 1: n]$ is obtained as direct combination with covariance inverses:

$$
\begin{array}{r}
P_{s}^{-1}[k \mid 1: n]= \\
\hat{x}_{s}[k \mid 1: n]=P_{f}^{-1}[k \mid 1: k]+P_{b}^{-1}[k \mid k+1: n]\left(P_{f}^{-1}[k \mid 1: k] \hat{x}_{f}[k \mid 1: k]\right. \\
\\
\left.+P_{b}^{-1}[k \mid k+1: n] \hat{x}_{b}[k \mid k+1: n]\right)
\end{array}
$$

So, this forward-backward combination implements the optimal smoother (if models are correct), the estimate at time $k$ conditioned to all available observations from 0 to $n$.

\section{Results}

This section describes some results to exemplify the segmentation and reconstruction accuracies comparing them to classical approaches in the literature. They were obtained from a synthetic evaluation scenario containing 57 trajectories in representative ATC conditions, eleven radars (PSR/SSR and Mode-S) with different scan periods of 12 and $4 \mathrm{~s}$, and noises in the measured range and azimuth with standard deviations of $\sigma_{r}=100 \mathrm{~m}, \sigma_{\theta}=0.09^{\circ}$, respectively. In this data set, 30 of the 57 simulated trajectories included the condition of aircraft equipped with ADD capability to send groundspeed and heading measurements from on-board sensors (in addition to the radar data), allowing the described enrichments of the reconstruction process. The scenario contains four groups of trajectories (see Fig. 3), which change in magnitudes of acceleration, the relative positions with respect to sensors (radial, tangential) and distances:

- Uniform Motion (22): variations in heading time length, relative position.

- Uniform-Turn-Uniform (17): varying transversal accelerations from 2.5 to $6 \mathrm{~m} / \mathrm{s}^{2}$.

- Uniform-Accel-Uniform (12): varying longitudinal acceleration from 0.5 to $1.2 \mathrm{~m} / \mathrm{s}^{2}$.

- Racetrack trajectories (6): turns at $4 \mathrm{~m} / \mathrm{s}^{2}, 150 \mathrm{~m} / \mathrm{s}$ of speed and average distance of $100 \mathrm{~km}$.
Both the simulation and reconstruction algorithms are implemented for analysis in a mock-up platform in $\mathrm{C}++$ developed by authors, and the final version is contained in EUROCONTROL SASS-C v7 system.

To detail the performance of our algorithms we select one of the racetrack trajectories, depicted in Fig. 4. It contains a series of alternated turns and uniform segments with the shape of a squared hippodrome. Turns are of $90^{\circ}$, with $4 \mathrm{~m} / \mathrm{s}^{2}$ transversal acceleration. The figure also contains the WLS estimated parameters of the maneuvers; the magenta inner circles have been drawn with the estimated center and radio of the four turning maneuvers.

The algorithm correctly segmented this trajectory in four segments, as the example presented in [36]: the forward-backward probabilities are used to identify the segments, only with a few errors in the location of edges. The confusion matrix is presented in Table 2, compared with a previous approach where the probabilities are directly combined [18] (numbers are percentages, and two confusion matrices are presented together, corresponding to IMM and MBR).

Fig. 5 shows how the MoF-oriented IMM (first step of MBR) works, illustrating the effect of oriented covariance matrices which affect to mode residuals and so to the corresponding mode probabilities $\left(\mu_{i}[k]\right)$. The uniform mode alternates with the transversal mode, continuous lines are used to print forward run and dotted lines for backward, with different colors for mode (uniform " 1 " in blue, turn " 2 " in red, longitudinal " 3 " in magenta).

In Figs. 6-9, the reconstruction errors are compared for three reconstruction techniques: the proposed model-based IMM reconstruction (MBR), the IMM smoothing by Helmick et al. [18] (including in the output both smoothed states and mode probabilities after Bayesian combination of forward-backward runs) (IMM), and a beta-spline reconstruction, based on the smoothing cubic spline available in the MATLAB ${ }^{\mathrm{TM}}$.

The behavior of the spline approach is the most reactive to measurements and also the most affected by noise, so the average power of residual noise is very high compared with the other techniques. The reason is the fixed length of time window to obtain the smoothed curve with a spline, which limits its filtering effect with respect to the recursive techniques. The IMM smoother and MBR have equivalent performance in uniform segments, while the advantage of MBR is evident both in transitions and during maneuvering segments, where the use of more accurate prediction models and appropriate transition matrices reduces the

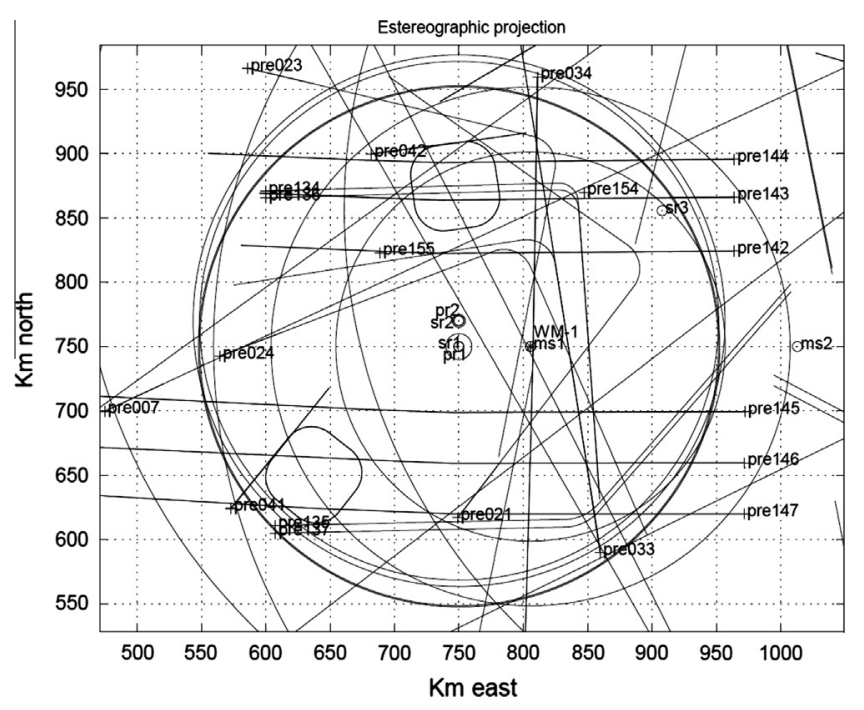

Fig. 3. Simulated synthetic scenario. 


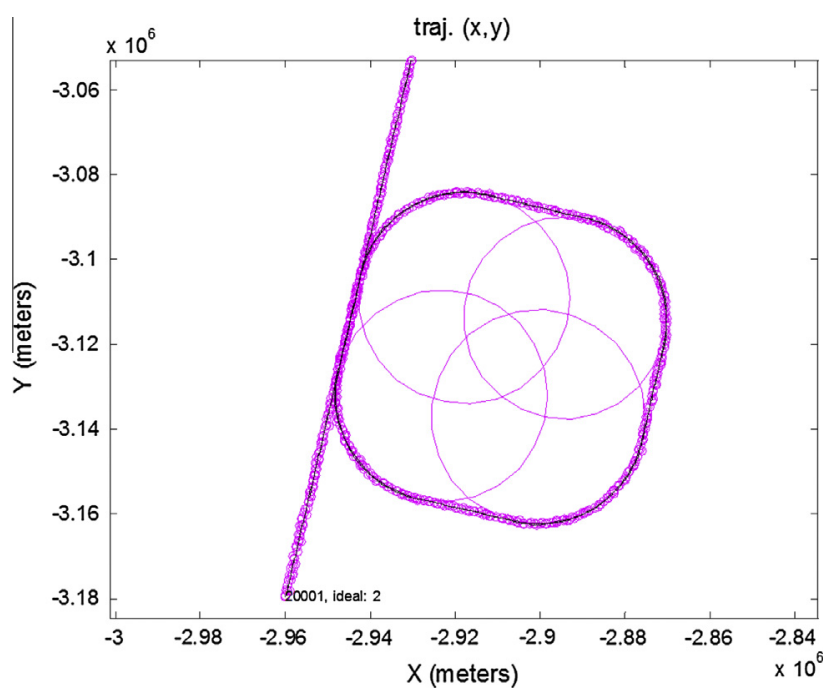

Fig. 4. Reconstruction in racetrack trajectory ( $4 \mathrm{~m} / \mathrm{s}^{2}$ acceleration).

Table 2

MoF confusion matrices for the racetrack trajectory. IMM-Smoother probabilities [18] vs MBR classification.

\begin{tabular}{llllll}
\hline \multicolumn{2}{l}{ Declared MoF (\%) } & \multicolumn{3}{l}{} \\
\hline \multicolumn{2}{l}{ True MoF } & $\mathrm{M}_{1}$ & $\mathrm{M}_{2}$ & $\mathrm{M}_{3}$ & Unknown \\
$\mathrm{M}_{1}$ & $\mathrm{IMM}$ & 48.97 & 10.18 & 0 & 0 \\
& $\mathrm{MBR}$ & 58.85 & 0.29 & 0 & 0 \\
$\mathrm{M}_{2}$ & $\mathrm{IMM}$ & 5.01 & 35.84 & 0 & 0 \\
& $\mathrm{MBR}$ & 1.92 & 38.94 & 0 & 0 \\
$\mathrm{M}_{3}$ & $\mathrm{IMM}$ & 0 & 0 & 0 & 0 \\
& $\mathrm{MBR}$ & 0 & 0 & 0 & 0 \\
\hline
\end{tabular}

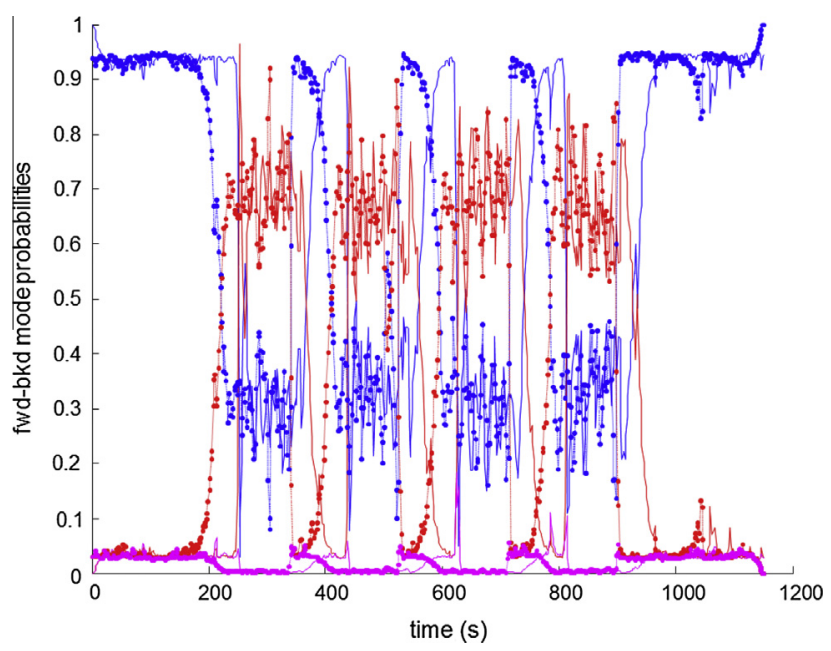

Fig. 5. Probability modes in forward and backward runs, racetrack trajectory (continuous fwd, dotted bkd).

maneuver-induced bias in the transitions and during turn and, simultaneously, apply a less noisy reconstruction process in segments classified and validated as turns.

Similar conclusions can be extracted for reconstruction accuracy with the complete scenario. Figs. 10 and 11 visually summarize the error distributions for position (across-track component) and velocity (groundspeed) along all trajectories, containing the 50\% (boxplot) and 95\% (whisker) confidence intervals of all available data.

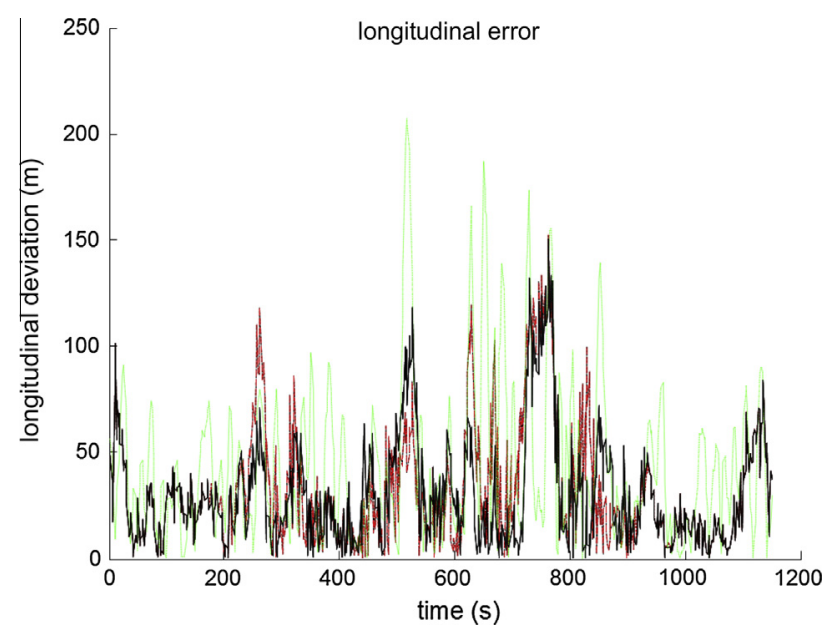

Fig. 6. Longitudinal error with simulated racetrack (black - MBR, red --- IMM, green …... spline). (For interpretation of the references to colour in this figure legend, the reader is referred to the web version of this article.)

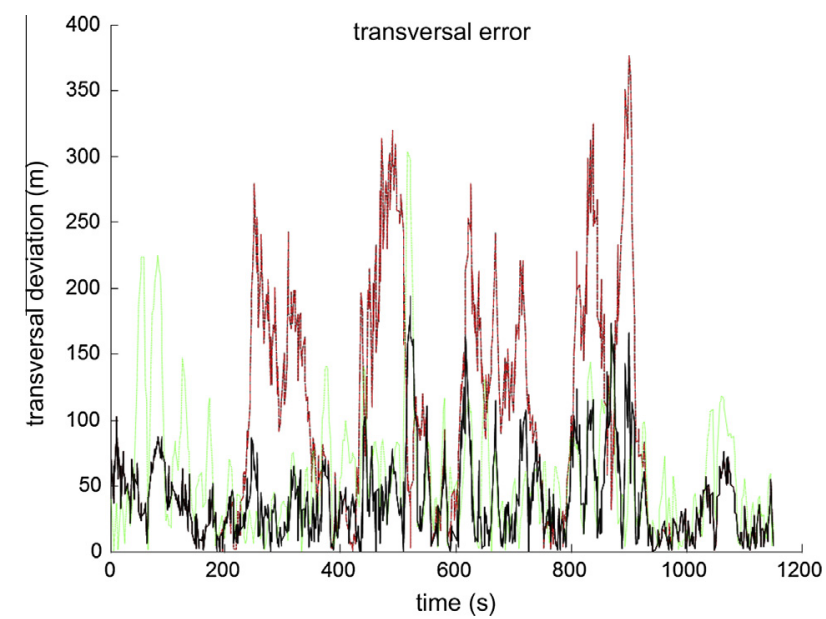

Fig. 7. Transversal error with simulated racetrack (black - MBR, red --- IMM, green .... spline). (For interpretation of the references to colour in this figure legend, the reader is referred to the web version of this article.)

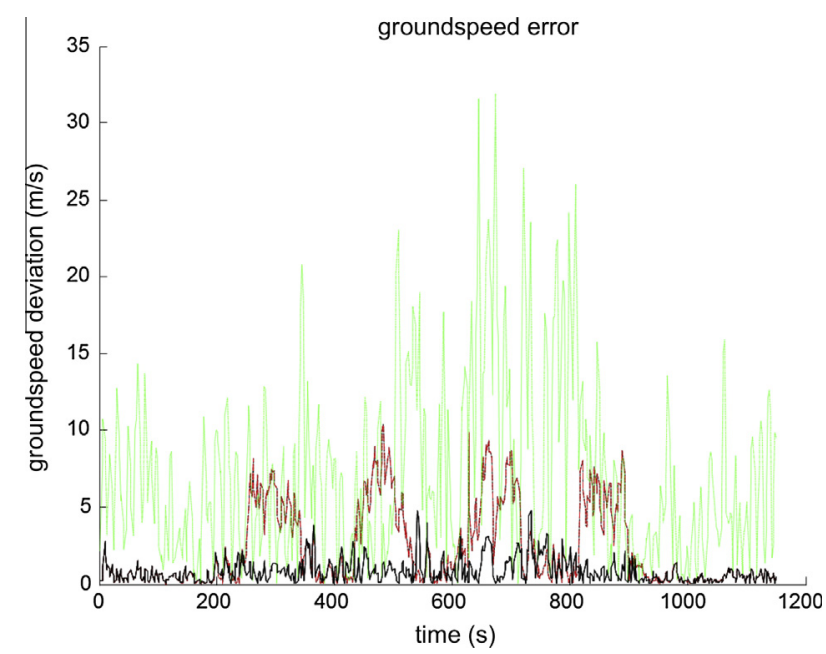

Fig. 8. Groundspeed error with simulated racetrack (black - MBR, red --- IMM, green …... spline). (For interpretation of the references to colour in this figure legend, the reader is referred to the web version of this article.) 


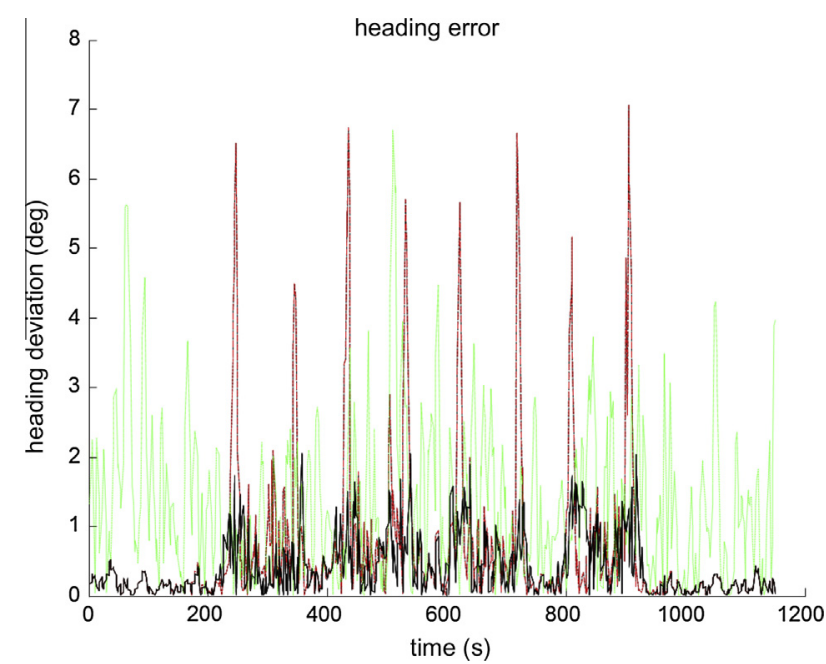

Fig. 9. Heading error with simulated racetrack (black - MBR, red --- IMM, green ..... spline). (For interpretation of the references to colour in this figure legend, the reader is referred to the web version of this article.)

Table 3 contains the corresponding root mean squared values grouped accordingly to the three types of MoF for all trajectories. The conclusion of quantitative analysis is quite clear, indicating significant advantage of MBR over fixed IMM smoothing especially during maneuvering segments. The limitation of Beta-spline due to its short-term noise filtering is also evident from the distribution figures and quadratic errors.

The use of aircraft derived data with groundspeed and heading lead to further improvement in reconstruction. It also provided advantage of classification over all trajectories in the simulated scenario considering three alternatives (see Table 4 with percentages corresponding to the three confusion matrices): position-only IMM probabilities and edge detection, MBR rules with position measurements, and a combination of segments extracted from position and ADD velocities ("MBR-Pos+vel" in table). The classification obtained with IMM probability smoothing (only position) [18], is the reference for comparison in the first place (rows $1,4,7$ ). Numbers are percentages for the three confusion matrices presented together, corresponding to IMM and MBR-position and MBR-position+velocity. The rates of success (diagonal of the three confusion matrices) are later presented in Table 5.

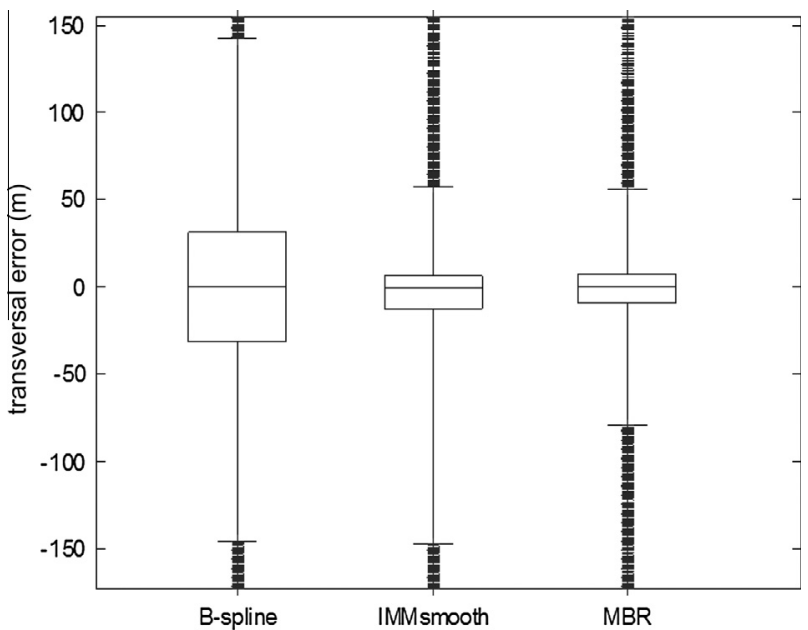

Fig. 10. Transversal error in all trajectories. MBR, B-spline and IMM comparison.

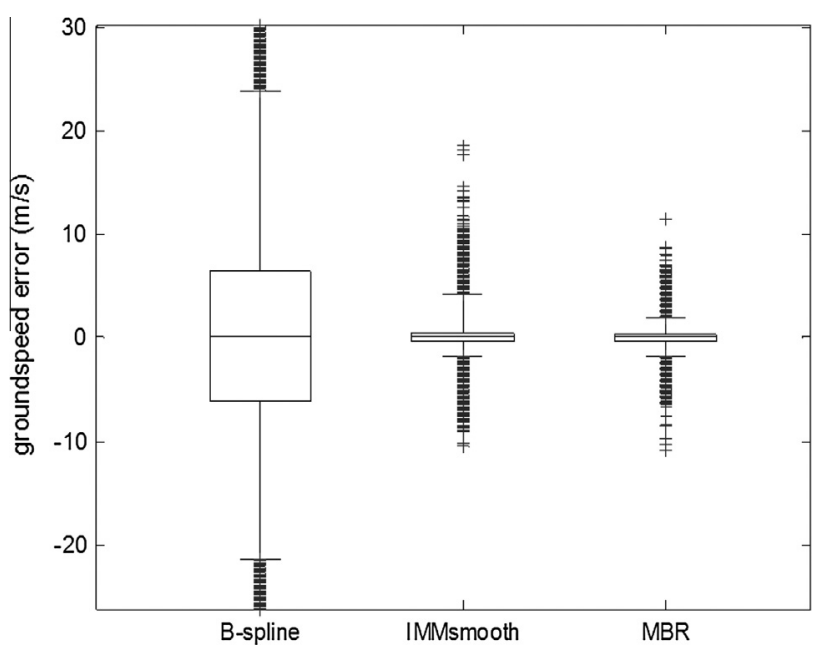

Fig. 11. Groundspeed error in all trajectories. MBR, B-spline and IMM comparison.

Table 3

Reconstruction errors (RMS values) in the whole simulated scenario.

\begin{tabular}{lccc} 
& Beta-spline & IMM & MBR \\
\hline Transversal error $(m)$ & & & \\
$\mathrm{M}_{1}$ & 77.0 & 33.4 & 33.1 \\
$\mathrm{M}_{2}$ & 64.2 & 135.7 & 44.5 \\
$\mathrm{M}_{3}$ & 40.2 & 14.5 & 13.8 \\
Average & 74.3 & 51.2 & 33.5 \\
Longitudinal error $(m)$ & & & \\
$\mathrm{M}_{1}$ & 67.3 & 26.1 & 25.7 \\
$\mathrm{M}_{2}$ & 67.2 & 41.8 & 32.8 \\
$\mathrm{M}_{3}$ & 41.2 & 26.1 & 14.6 \\
Average & 66.1 & 27.9 & 25.9 \\
Groundspeed error $(m / s)$ & & \\
$\mathrm{M}_{1}$ & 11.0 & 0.82 & 0.8 \\
$\mathrm{M}_{2}$ & 11.6 & 3.5 & 1.3 \\
$\mathrm{M}_{3}$ & 9.3 & 2.1 & 1.2 \\
Average & 11.0 & 1.4 & 0.9 \\
Heading error $\left(^{\circ}\right)$ & & & \\
$\mathrm{M}_{1}$ & 2.7 & 0.19 & 0.24 \\
$\mathrm{M}_{2}$ & 2.0 & 1.8 & 0.84 \\
$\mathrm{M}_{3}$ & 1.6 & 0.11 & 0.13 \\
Average & 2.6 & 0.59 & 0.34 \\
\hline & & &
\end{tabular}

Analogously, the impact of ADD information over reconstruction accuracy with MBR is summarized in Table 6 with the average squared errors per MoF category, which can be compared with results in Table 3.

\section{Vertical smoothing summary}

In this section we will include a summarized description of vertical smoothing for completeness. It should be noted that there are different kinds of height sources, some providing barometric height information (Mode C, ADS-B in some cases), and others providing geometric height (WAM, 3D primary radar, ADS-B in other cases).

Vertical smoothing is also based on several steps, with a functional decomposition similar to the one in horizontal processing:

- Vertical segmentation.

- Vertical segments validation.

- Vertical smoothing with protection against overshooting. 
Table 4

MoF confusion matrices for the simulated scenario, with IMM and MBR, with and without velocities.

\begin{tabular}{|c|c|c|c|c|c|}
\hline \multicolumn{6}{|c|}{ Declared MoF (\%) } \\
\hline & & $\mathrm{M}_{1}$ & $\mathrm{M}_{2}$ & $\mathrm{M}_{3}$ & Unknown \\
\hline \multicolumn{6}{|c|}{ True MoF } \\
\hline \multirow[t]{3}{*}{$\mathrm{M}_{1}$} & IMM & 83.23 & 1.46 & 0.63 & 0 \\
\hline & MBR-position & 84.43 & 0.10 & 0.30 & 0.49 \\
\hline & MBR-Pos+vel & 84.23 & 0.33 & 0.78 & 0 \\
\hline \multirow[t]{3}{*}{$\mathrm{M}_{2}$} & IMM & 0.86 & 8.17 & 0.01 & 0 \\
\hline & MBR-position & 1.48 & 7.28 & 0.01 & 0.26 \\
\hline & MBR-Pos+vel & 0.21 & 8.80 & 0 & 0.01 \\
\hline \multirow[t]{3}{*}{$\mathrm{M}_{3}$} & IMM & 1.35 & 0.01 & 4.28 & 0 \\
\hline & MBR-position & 0.76 & 0 & 4.76 & 0.12 \\
\hline & MBR-Pos+vel & 0.27 & 0 & 5.37 & 0 \\
\hline
\end{tabular}

Table 5

Global classification success rate.

\begin{tabular}{ll}
\hline Global success rate (\%) & \\
\hline 95.676 & IMM \\
96.474 & MBR-position \\
98.405 & MBR-Pos+vel \\
\hline
\end{tabular}

Table 6

Reconstruction errors (RMS values), impact of velocity information on MBR algorithm.

\begin{tabular}{ll}
\hline & MBR-pos+vel \\
\hline Transversal error $(m)$ & 31.2 \\
$\mathrm{M}_{1}$ & 41.3 \\
$\mathrm{M}_{2}$ & 10.2 \\
$\mathrm{M}_{3}$ & 31.5 \\
Average & \\
Longitudinal error $(m)$ & 20.9 \\
$\mathrm{M}_{1}$ & 27.2 \\
$\mathrm{M}_{2}$ & 12.1 \\
$\mathrm{M}_{3}$ & 21.2 \\
Average & \\
Groundspeed error $(m / s)$ & \\
$\mathrm{M}_{1}$ & 0.43 \\
$\mathrm{M}_{2}$ & 0.83 \\
$\mathrm{M}_{3}$ & 0.60 \\
Average & 0.49 \\
Heading error $\left(^{\circ}\right)$ & \\
$\mathrm{M}_{1}$ & 0.31 \\
$\mathrm{M}_{2}$ & 0.90 \\
$\mathrm{M}_{3}$ & 0.12 \\
Average & 0.39 \\
\hline
\end{tabular}

The vertical MoF segmentation is based on forward and backward vertical rate (velocity) estimates computed from height measurements. Those vertical velocity estimates are derived through a pair of Kalman filters, each of them with two states: height and vertical rate [17]. It is carried out independently for the both types of height sources, barometric and geometric, if available, so that we can have up to four velocity estimates (forward and backward for geometric and barometric height). After, the resulting vertical MoF segments are obtained by combination of segments obtained for each type of height. The segmentation process searches for segments with significant values of vertical velocity in forward and backward passes of Kalman filters, by comparing the estimated vertical rate against a constant threshold, to obtain the segment edges. Fig. 12 illustrates it with an example.

The vertical segmentation procedure then merges the segments from backward and forward filters by requesting detection on both filters for any given instant to make it belong to the resulting



Fig. 12. Illustration of vertical MOF segmentation with forward-backward filters.

vertical manoeuvre segment (AND logic). Finally, segments derived from barometric and geometric filters are also merged, when available, so that if a certain time interval is detected as belonging to a vertical manoeuvre by the segmentation process associated to any of the types of height, it is assumed to be part of a vertical manoeuvre interval (OR logic).

The rest of the processes are performed independently for barometric and geometric measurements, so that two reconstructions are finally derived: a barometric vertical height/rate reconstruction and a geometric vertical height/rate reconstruction. This is done in order to avoid potential problems in the reconstructions due to the presence of non-constant offset between both types of height information and to increase overall system robustness. So, the remaining of the process is done twice, once for each type of measurement.

In an analogous way to horizontal computation, a posterior refinement process for vertical segments is applied. It searches for very short segments, to be fused with their adjacent segments, as illustrated in Fig. 13. A one-dimensional version of the quality statistic described in Section 4 is used to check if fusing those

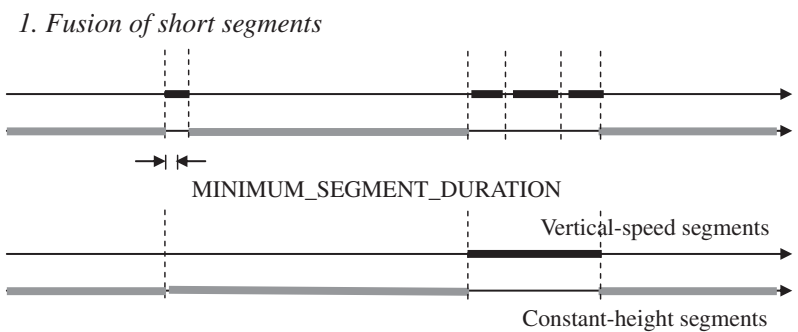

1. Fusion of short adjacent segments at constant height or vertical speed

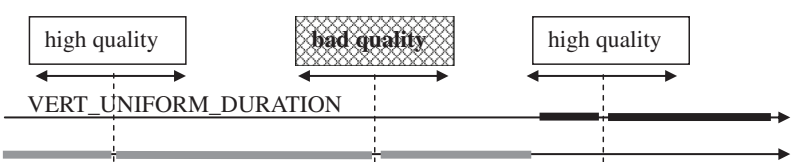

Vertical-speed segments

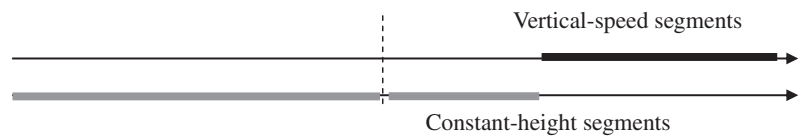

Fig. 13. Phases of vertical MOF segment refinement. 

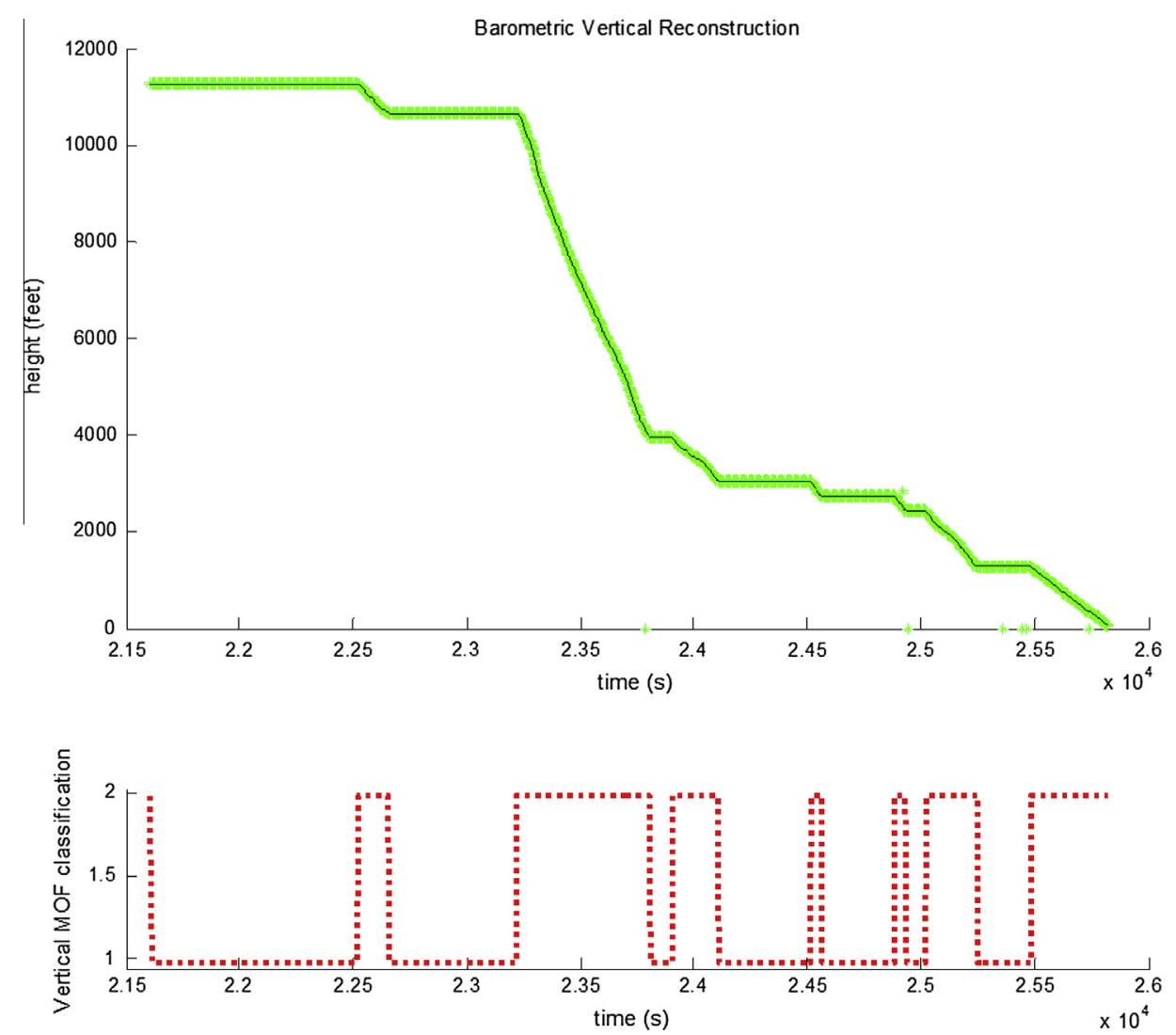

Fig. 14. Vertical reconstruction and MoF segmentation.
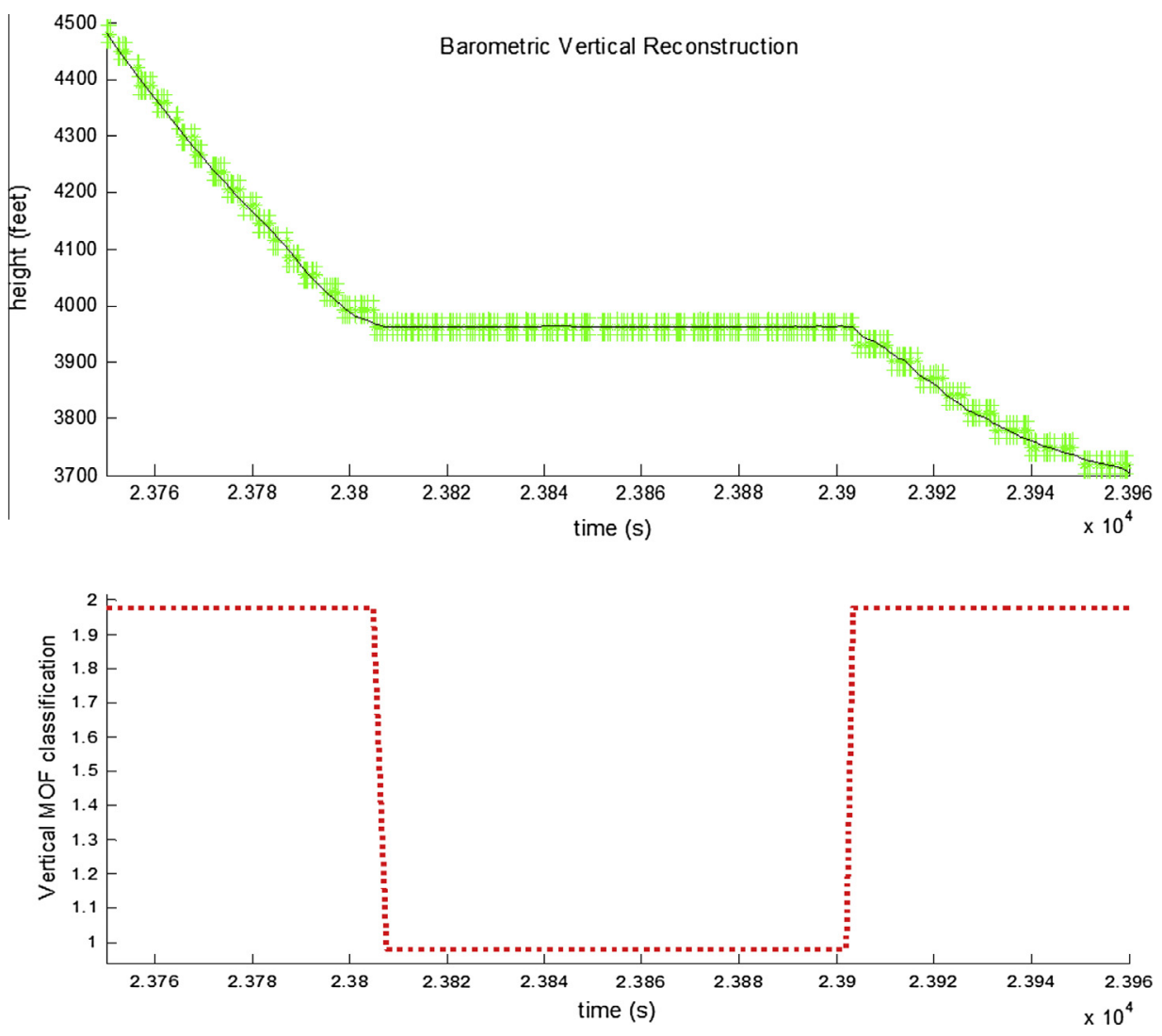

Fig. 15. Detail of transitions in vertical reconstruction. 
segments is feasible or not, by comparing its value to a given threshold for the fused segment. To do so, two different kinds of segments are defined:

- Constant height segments. They may be interpolated by a constant height value.

- Constant vertical rate segments. They may be interpolated by a constant slope function, with an initial height parameter.

Before making any merging of adjacent segments removing intermediate segments the new resulting segment residual (accumulated divergence between the interpolation and the measurements) is compared with a threshold, and if it is not consistent with the model the merging is discarded. For instance, in the second part of 13 , five different segments are processed. The first pair (from the left) has high quality (reduced residual with a same constant height) and therefore gets merged. Then, the merging of the two initial constant height segments with the following constant height segment is discarded as the quality measurement is bad, as there is no a constant height compatible with both segments (for instance, due to a small flight level change). Obviously, constant height and vertical speed segments cannot be merged. Then, the fourth and fifth segments (both marked as vertical speed), get merged as we are able to estimate a pair of interpolation parameters compatible with both segments measurements.

Finally, the smoothing process to compute vertical vectors (height and vertical velocity), is analogous to the horizontal reconstruction: a pair of forward-backward adaptive Kalman filters, with height and vertical speed states. There are several differences with respect to horizontal smoothing:

- Each of the filters has a time changing plant noise covariance matrix, so that it has low values in the middle of the segments and higher values near their edges, to be able to reduce the impact of the ongoing changes od modes of flight in the vertical trajectory reconstruction.

- The combination of the backward and forward filters takes into account now the available vertical MoF intervals instead of applying a direct combination with the same weights for every time. So, when a change of vertical MoF is close in time, the filtered estimated vector with no maneuver-induced bias (forward at the end of segments or backward at the beginning of segments) is selected in order to avoid combination with overshoot.

The process is illustrated with a real data reconstruction from a landing trajectory, in which the aircraft carries out some racetrack loops at constant height until the descent and approach to airport. Fig. 14 shows the vertical profile and result of segmentation process, encoded in two categories: "1" for constant height, " 2 " for descent segments. In green we have the measured heights along time, while in black we depict the associated smoothed trajectory.

In Fig. 15 we can see a time interval of the previously depicted descent trajectory in detail. The reconstruction is satisfactory, practically without overshoots. The vertical profile is also quite smoothed, computed from the barometric height which is affected by quantization in flight levels, as can be also appreciated in the figure.

\section{Conclusion}

This work presented a new approach for trajectory reconstruction based on the regular motion patterns present in ATC flights. The use of a model-based reconstruction technique for ATC trajectories provided satisfactory performance both for trajectory segmentation into homogeneous $\mathrm{MoF}$ segments and accurate reconstruction. The proposed algorithm is based on IMM smoothing, a technique with high efficiency for accurate estimation of maneuvering trajectories. The offline characteristic of this problem, together with the requirement of segmenting trajectories according to MoF, motivated the development of a variable reconstruction structure with modes and parameters conditioned to the sequence of MoF segments previously identified. Our proposal keeps multiple models whose behavior is conditioned to the computed MoF sequence. An independent validation mechanism (normalized residual averaging test over segments) is used to gain robustness, avoiding the use of bad parameters or assumptions in the reconstruction process.

The use of this model-based technique for reconstructing ATC trajectories increases accuracy compared with earlier fitting approaches. The performance results are clearly superior to betaspline smoothing, and also the advantage with respect to a conventional "fixed" IMM smoother is shown in the results. Future research will address the analysis of configuration parameters and opportunities for optimization with different approaches, and some mitigation procedures to deal with other realistic effects such as residual bias, outliers, irregular segments, appearing on operating conditions.

\section{Acknowledgments}

This work was supported in part by Projects EUROCONTROL TRES, MEyC TEC2012-37832-C02-01, MEyC TEC2011-28626-C0201/02 and CAM CONTEXTS (S2009/TIC-1485).

\section{References}

[1] J. Besada, A. Soto, G. de Miguel, J. García, E. Voet, ATC trajectory reconstruction for automated evaluation of sensors and trackers performance, IEEE AES Mag. 28 (2) (2013) 4-17.

[2] T. Kirubarajan, Y. Bar-Shalom, Precision large scale air traffic surveillance using IMM/assignment estimators, IEEE Trans. Aerosp. Electron. Syst. 35 (1) (1999) 255-266.

[3] W.H. Neven, H.A. Blom, C. de Kraker, Jump linear model based aircraft trajectory reconstruction, in: Proceedings of SPIE Conference on Signal and Data Processing of Small Targets, SPIE, vol. 2235, Orlando, 1994, pp. 540-557.

[4] A. Desmond-Kennedy, B. Gardner, Tools for analysing the performance of ATC surveillance radars. Specifying and measuring performance of modern radar systems, IEE Colloq. 221 (1998) 6/1-6/4.

[5] B. Ristic, N. Gordon, A. Bessell, On target classification using kinematic data, Inf. Fusion 5 (2004) 15-21.

[6] C. Lee, X. Yangsheng, Trajectory fitting with smoothing splines using velocity information, in: IEEE International Conference on Robotics and Automation, vol. 3, 2000, pp. 2796-2801.

[7] H.C. Lee, A phase space spline smoother for fitting trajectories, IEEE Trans. Syst., Man Cybernet., Part B 34 (1) (2004) 346-356.

[8] Q. Huang, S. Kajita, N. Koyachi, K. Kaneko, K. Yokoi, H. Arai, K. Komoriya, K. Tanie, A high stability, smooth walking pattern for a biped robot, in: Proceedings of the IEEE International Conference on Robotics and Automation, 1999, pp. 65-71.

[9] S. Takahashi, C.F. Martin, Optimal control theoretic splines and its application to mobile robot, in: Proceedings of the 2004 IEEE International Conference on Control Applications, 2004, pp. 1729-1732.

[10] C. de Boor, Applied Mathematical Sciences: A Practical Guide to Splines, vol. 27, Springer-Verlag, The Netherlands, 1978.

[11] J.J. Renes, P. Kraan, C. Eymann, Flightpath reconstruction and systematic radar error estimation from multi-radar range-azimuth measurements, in: Proceedings of 24th IEEE Conference on Decision and Control, 1985, pp. 1282-1285.

[12] W. Konle, Data Smoothing for Air Traffic Simulation. 6th Workshop on Sensor Data Fusion. Trends, Solutions, Applications, Berlin, Germany, 2011.

[13] M.O. St-Hilaire, E.H. Lefebvre, A. Christopher, Track modeling for maritime surveillance, in: Proceedings of the 2008 11th International Conference on Information Fusion, Cologne, Germany, 2008.

[14] Y. Wehn, R. Yates, P. Valin, A. Guitouni, E. Bossé, A. Dlugan, H.A. Zwick, A distributed information fusion testbed for coastal surveillance, in: Proceedings of 10th Annual Conference on Information Fusion, Quebec, Canada, 2007.

[15] B. Teixeira, L. Torres, P. Iscold, L. Aguirre, Flight path reconstruction - a comparison of nonlinear Kalman filter and smoother algorithms, Aerosp. Sci. Technol. 15 (2011) 60-71. 
[16] S. Shu-Li, Multi-sensor optimal fusion fixed-interval Kalman smoothers, Inf. Fusion 9 (2008) 293-299.

[17] A. Gelb, Applied Optimal Estimation, The MIT Press, Cambridge (MA), 1974.

[18] R.E. Helmick, W.D. Blair, S.A. Hoffman, Fixed-interval smoothing for Markovian switching systems, IEEE Trans. Inf. Theory 41 (6) (1995) 1845-1855.

[19] B. Chen, J.K. Tugnait, Interacting multiple model fixed-lag smoothing algorithm for Markovian switching systems, IEEE Trans. Aerosp. Electron. Syst. 36 (1) (2000) 243-250.

[20] W. Koch, Fixed-interval retrodiction approach to Bayesian IMM-MHT for maneuvering multiple targets, IEEE Trans. Aerosp. Electron. Syst. 36 (1) (2000) 2-14.

[21] Y. Bar-Shalom, Xiao-Rong Li, Estimation and Tracking. Principles, Techniques and Software, Artech House, 1993.

[22] X.R. Li, V.P. Jilkov, Survey of maneuvering target tracking. Part V: Multiple-model methods, IEEE Trans. Aerosp. Electron. Syst. 41 (4) (2005) 1255-1321.

[23] J. García, A. Soto, G. de Miguel, J. Besada, P. Tarrio, Trajectory reconstruction techniques for evaluation of ATC systems, Tyrrhenian International Workshop on Digital Communications - Enhanced Surveillance of Aircraft and Vehicles, Capri, Italy, 2008.

[24] J. Garcia, J.M. Molina, G. de Miguel, J.A. Besada, Model-based trajectory reconstruction using imm smoothing and motion pattern identification, in: 10th International Conference on Information Fusion, Quebec, Canada, 2007.

[25] J.A. Besada, G. de Miguel, A.M. Bernardos, J.R. Casar, Generic multisensor multitarget bias estimation architecture, IET Radar Sonar Navig. 6 (5) (2012) 365-378.

[26] H.A.P. Blom, Y. Bar-Shalom, The interacting multiple model algorithm for systems with Markovian switching coefficients, IEEE Trans. Autom. Control 33 (8) (1988) 780-783.

[27] S. Coraluppi, C. Carthel, Recursive track fusion for multi-sensor surveillance, Inf. Fusion 5 (2004) 23-33.

[28] S. Särkka, A. Vehtari, J. Lampinen, Rao-Blackwellized particle filter for multiple target tracking, Inf. Fusion 8 (2007) 2-15.

[29] T.L. Ogle, W.D. Blair, Fixed-lag alpha-beta filter for target trajectory smoothing, IEEE Trans. Aerosp. Electron. Syst. 40 (4) (2004) 1417-1421.
[30] H.A.P. Bloom, Y. Bar-Shalom, Time-reversion of a hybrid state stochastic difference system with a jump-linear smoothing application, IEEE Trans. Inf. Theory 36 (4) (1990) 836-847.

[31] W. Li, Y. Jia, Rao-Blackwellised particle filtering and smoothing for jump Markov non-linear systems with mode observation, IET Signal Proc. 7 (4) (2013) 327-336.

[32] H. Kamel, W. Badawy, A smoothing Rao-Blackwellized particle filter for tracking a highly-maneuverable target, in: Proceedings of 2005 IEEE International Radar Conference, 2005, pp. 967-970, Proceedings of 24th IEEE Conference on Decision and Control, 1985, pp. 1282-1285.

[33] P. Bunch, S. Godsill, Improved particle approximations to the joint smoothing distribution using Markov chain Monte Carlo, IEEE Trans. Signal Process. 6 (4) (2013) 956-963.

[34] Mélanie Bocquel, Multitarget tracking with multiscan knowledge exploitation using sequential MCMC sampling, IEEE J. Sel. Top. Signal Process. 7 (3) (2013) 534-542.

[35] E. Keogh, S. Chu, D. Hart, M. Pazzani, Segmenting Time Series: A Survey and Novel Approach, Data Mining in Time Series Databases, second ed., World Scientific, New York, 2003.

[36] L. Xiaoyan, Z. Lin, H. Wang, Novel online methods for time series segmentation, IEEE Trans. Knowl. Data Eng. 20 (12) (2008) 1616-1626.

[37] J. García, O. Pérez, J.M. Molina, G. de Miguel, Trajectory classification based on machine-learning techniques over tracking data, in: Proceedings of 9th International Conference on Information Fusion, Florence, Italy, 2006.

[38] T. Kariya, H. Kurata, Generalized Least Squares, Wiley, New York, 2004.

[39] J. García, J.A. Besada, J.R. Casar, Use of map information for tracking targets on airport surface, IEEE Trans. Aerosp. Electron. Syst. 39 (2) (2009) 675-694.

[40] J.A. Besada, J. García, G. de Miguel, A. Berlanga, J.M. Molina, J.R. Casar, Design of IMM filter for radar tracking using evolution strategies, IEEE Trans. Aerosp. Electron. Syst. 41 (3) (2005) 1109-1122.

[41] J. García, J.A. Besada, A. Berlanga, J.M. Molina, G. de Miguel, J.R. Casar, Application of evolution strategies to the design of tracking filters with a large number of specifications, EURASIP J. Appl. Signal Process. 8 (2003) 766-779. 